\title{
Casas consistoriales en Cundinamarca. Estudio de caso de la casa del municipio de Cogua
}

\author{
Claudia Uribe Kaffure \\ Cecilia Teresa López Pérez \\ Daniel Mauricio Ruiz Valencia
}

Historia

Los primeros asentamientos españoles en América se desarrollaron a partir de un trazado en damero y una plaza principal que daban origen a la organización urbana y política. La nueva ciudad reunía a pobladores españoles e indígenas, recibía un nuevo nombre, se repartían los solares y se establecía el cabildo. Este último era la institución colegiada establecida por la corona española para la administración de ciudades y villas, y el órgano por medio del cual los vecinos velaban por la adecuada atención de los problemas judiciales, administrativos, económicos y militares de la ciudad (Hernández, 2002; Rubio, 2006; Gutiérrez, 1990).

El primer cabildo era nombrado por los descubridores y los regidores elegían a quienes habrían de sucederles. En el siglo XV1, con la Real Cédula del 14 de diciembre de 1606, Felipe 11 estableció el régimen de venta de cargos al mejor postor, por remate de los oficios municipales, con el objeto de generar nuevas fuentes de ingresos para la corona española (Hernández, 2002; Wyrobisz, 1980). El comprador obtenía un título perpetuo que era transmisible pagando de nuevo una parte del valor. Esta figura dio origen a una élite urbana, a menudo con relaciones de parentesco, que usó sus cargos para velar por sus propios intereses (Mayorga, 2002).

La estructura y composición del cabildo tomó el modelo español, pero sus facultades variaban dependiendo de las características de la sociedad colonial en que se estableciera (Mayorga, 2002). Paralelos a estos se crearon los cabildos eclesiásticos, a los que les correspondía velar por la implantación de la fe católica en el nuevo reino.

El cabildo, como ente institucional, debía reunirse de forma regular y las sesiones se celebraban en la casa consistorial con la presencia del oidor, el gobernador, el teniente o el alcalde asignado para presidirla (Mayorga, 2002; Rubio, 2006).

La casa consistorial, entonces, era la casa de la villa o ciudad donde se reunían los capitulares de un ayuntamiento para celebrar sus juntas "senatoria domus, domus pretoriana" (RAE, 1791). A esta

Cómo citar este artículo: Uribe, C., López, C. y Ruiz, D. (2014). Casas consistoriales en Cundinamarca. Estudio de caso de la casa del municipio de Cogua. Apuntes, 27(1), 124-141. http://dx.doi.org/10.11144/Javeriana.APC27-1.ccce 


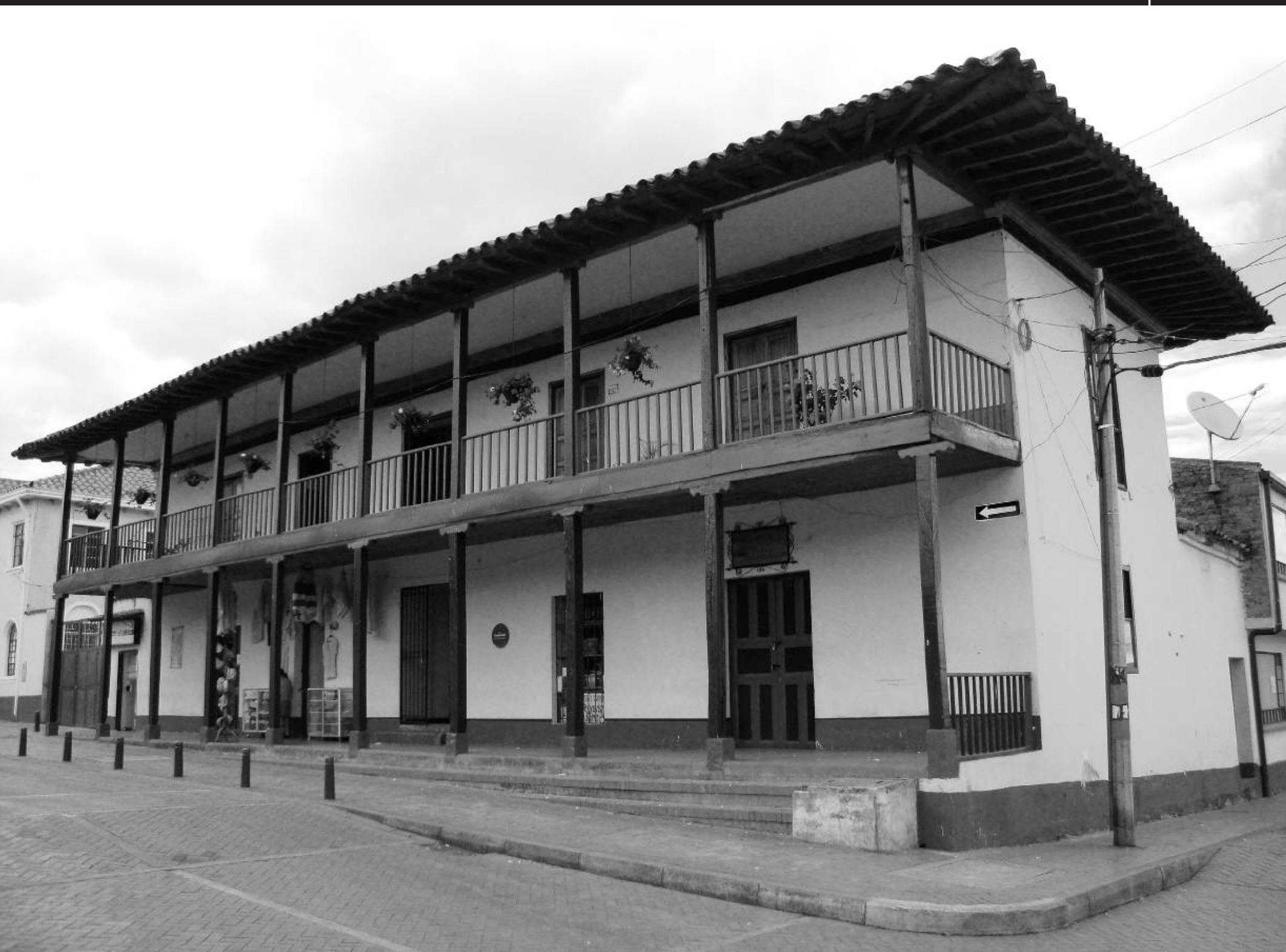

Casa Consistorial Cogua, Cundinamarca. Fuente:

Elaboración propia 


\section{Casas consistoriales en Cundinamarca. Estudio de caso de la casa del municipio de Cogua}

Town halls in Cundinamarca. Case Study of the town hall in the municipality of Cogua

Casas consistoriais em Cundinamarca. Estudo de caso da casa do município de Cogua

\section{Claudia Uribe Kaffure}

Docente de la Universidad del Tolima. Estudiante de Maestría en Ingeniería Civil, Pontificia Universidad Javeriana. Perteneciente al grupo de investigación Riesgos en sistemas naturales y antrópicos, de la Pontificia Universidad Javeriana, sede Bogotá

Cecilia Teresa López Pérez

Profesor Asistente, Departamento de Arquitectura, Pontificia Universidad Javeriana.

Daniel Mauricio Ruiz Valencia

Profesor Asistente, Departamento de Ingenieria civil, Pontificia Universidad Javeriana

\section{Resumen}

Con la creación de las primeras poblaciones en América nacen las instituciones encargadas de administrar las ciudades y velar por la adecuada solución de los problemas judiciales, administrativos y económicos de sus pobladores. Estas instituciones, que fueron creadas por los españoles, recibieron el nombre de cabildo. El lugar donde se reunía periódicamente a sesionar el cabildo recibía el nombre de casa consistorial, casa municipal, casa de gobierno o casa de ayuntamiento. En este artículo se presenta un breve recuento de las casas consistoriales de la época colonial que aún están en pie en el departamento de Cundinamarca. Se describe su tipología desde el punto de vista arquitectónico y estructural, mediante el estudio de caso específico de la casa consistorial de Cogua. Igualmente se estudia la respuesta de la edificación ante cargas de servicio y posibles eventos sísmicos y se propone un reforzamiento que mejore su desempeño estructural.

Palabras clave: casas consistoriales; ayuntamiento; historia; comportamiento estructural

Abstract

With the creation of the first American settlements, the institutions responsible for managing cities and ensuring the proper settlement of the legal, administrative and economic problems of its inhabitants are born. These institutions, which were created by the Spanish, were called councils. The place where they met regularly to session the council was called the Town Hall, Municipal house, government house or guildhall. This article provides a brief overview of the town halls of the colonial era that are still standing in the department of Cundinamarca. Its typology is described in terms of architectural and structural terms, by studying the specific case of the town hall of Cogua. Likewise it is studied the response of the building to service loads and potential seismic events and it is proposed a reinforcement to improve its structural performance.

Keywords: town halls; City Hall; history; structural performance

\section{Resumo}

Com a criação dos primeiros povoados na América nascem as instituições encarregadas de administrar as cidades e vigiar a adequada solução dos problemas judiciais, administrativos e económicos dos povoadores. Estas instituições, que foram criadas pelos espanhóis, receberam o nome de cabildo. 0 local onde reunia-se periodicamente para o cabildo fazer sessões recebia o nome de casa consistorial, casa municipal, casa de governo ou casa de ayuntamiento (prefeitura). Neste artigo apresenta-se breve reconto das casas consistoriais da época colonial que ainda ficam de pé no departamento de Cundinamarca. Descreve-se sua tipologia desde o ponto de vista arquitetônico e estrutural, mediante estudo de caso específico da casa consistorial de Cogua. Igualmente estuda a resposta da edificação ante cargas de serviço e possíveis eventos sísmicos e propõe-se reforço para melhorar seu desempenho estrutural.

Palavras-chave: casas consistoriais; prefeitura; história; comportamento estrutural

\section{octubre de 2013}

Aceptación: 25 de noviembre de 2013

Disponible en línea: 2 de enero de 2014 doi: 10.11144/Javeriana.APC27-1.ccce 
edificación se le conoce con diferentes nombres como: casa del ayuntamiento (en España), casa municipal, casa del cabildo, casa de gobierno y casa capitular. Esta última, llamada así por incluir dentro de sus instalaciones la sala capitular (Mayorga, 2002).

Estas edificaciones eran escenario de los sucesos y decisiones políticas más importantes del lugar. Sin embargo, José Manuel Groot (18001887) señala que allí también se llevaban a cabo actividades de carácter cultural.

En el caso de Bogotá, Hettner (1976) menciona que en el primer piso funcionaban almacenes y en el segundo la administración municipal. Durante las épocas de fiestas y eventos urbanos los balcones eran usados por las autoridades y la élite de la ciudad para observar los espectáculos y eventos de carácter religioso (Montoya, 2007).

A partir de la independencia de las diferentes naciones en lberoamérica, se nombraron juntas de gobierno, independientes del gobierno español, que establecieron sus propias constituciones, y cambiaron su organización en cantones, provincias y distritos parroquiales que nombraban sus funcionarios, alcaldes y jefes políticos (Hernán-
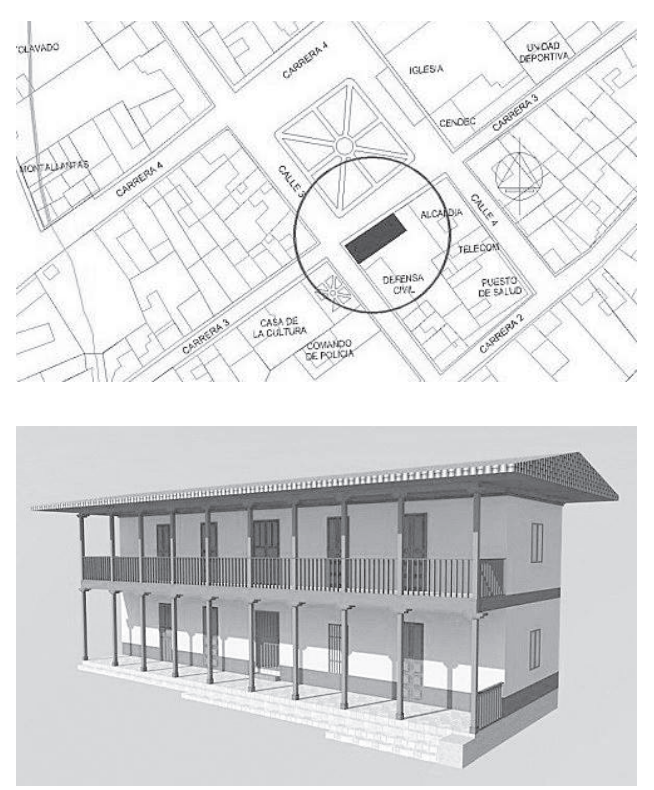

dez, 2002; López, 2006); sin embargo, las casas de gobierno se conservaron en los lugares originalmente establecidos por los españoles.

En América se reporta el desarrollo de estas construcciones en México: Chiapas, Queretaro, (lbarra, 2003; Hernández, 2013; Esponda, 2010), Cuba (Moreno y Marrero, 2008) y en Perú (Sánchez, 2003).
Figura 1:

a) Ubicación de la casa consistorial de Cogua. Enmarcada en una esquina de la plaza principal del pueblo b) casa consistorial de Cogua

Fuente: elaboración propia
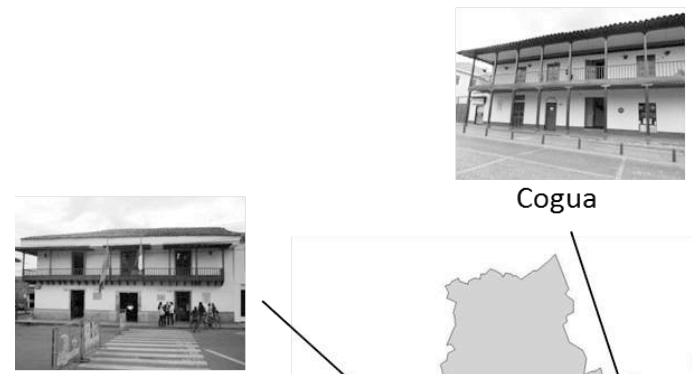

Funza

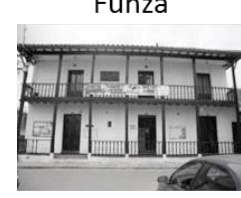

Madrid

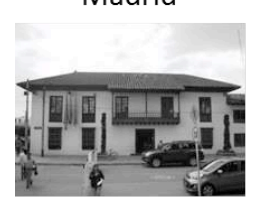

Mosquera

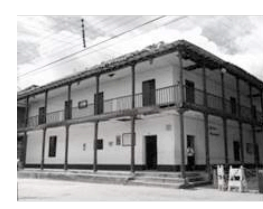

Agua de Dios

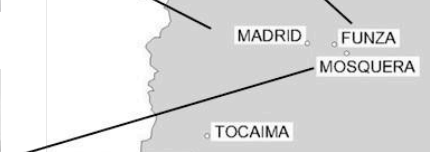

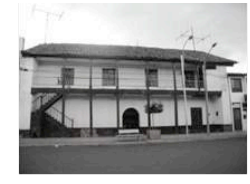

Simijaca
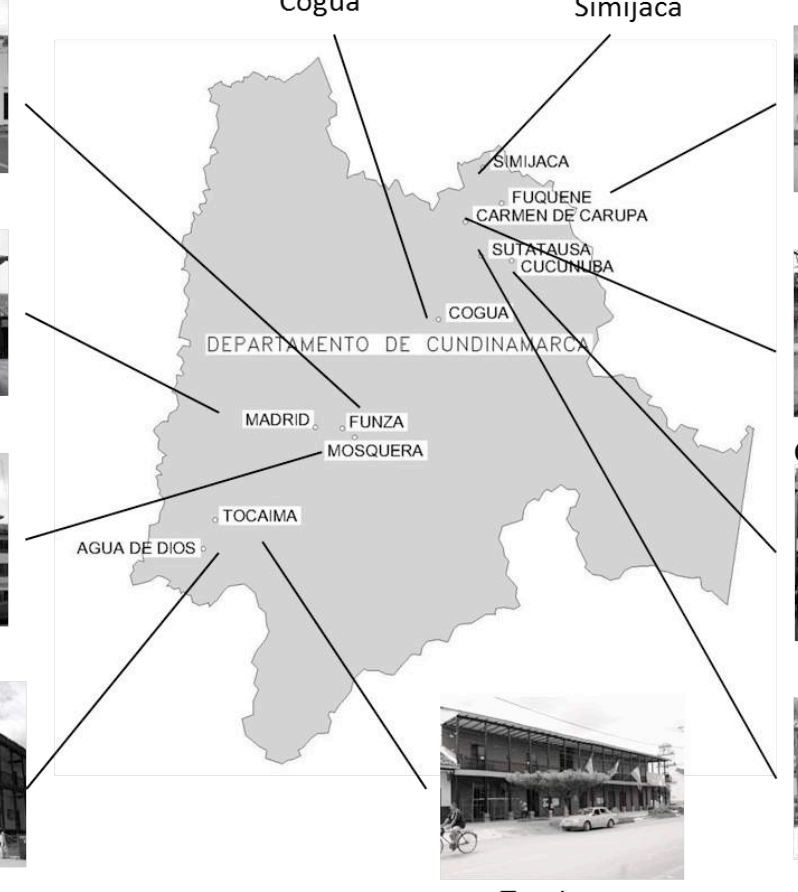

Tocaima

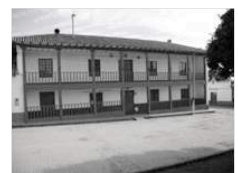

Fuquene

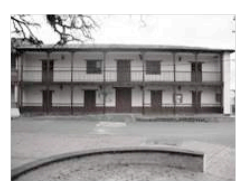

Carmen de Carupa
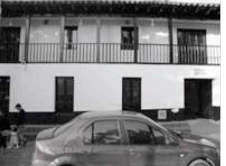

Cucunuba

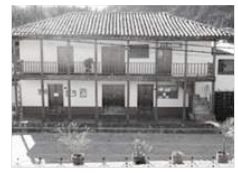

Sutatausa
Figura 2:

Casas consistoriales en Cundinamarca. Se observa la gran similitud entre las diferentes edificaciones de la región Fuente:

elaboración propia 
Cuadro 1: Casas consistoriales en Cundinamarca

\begin{tabular}{|l|l|}
\hline Casas consistoriales en 1886 & Otras casas consistoriales \\
\hline Usme, Chipaque, Une, Fosca, Cáqueza, Quetame, & Sutatausa, Fúquene, Simijaca, Carmen de \\
Fómeque, Choachí, Facatativá, Madrid, Mosquera, & Carupa, Cucunubá, Cogua, y Agua de Dios \\
Funza, Cota, Suba, Engativá, Bogotá, Guaduas, & \\
Tocaima, Chocontá, Guateque y Ubaque & \\
\hline
\end{tabular}

Fuente: elaboración propia

En Colombia se encuentran en Soledad, en el departamento de Atlántico (Solano, 2009), Santa

Figura 3: Casa consistorial de Chipaque en

la actualidad

Fuente:

elaboración propia

Fe de Antioquia, en Antioquía (Cordero, 2011), Cartagena, en Bolívar (Solano, 2010), Pradera, Sevilla y Caicedo, en el Valle del Cauca, y las descritas por Rufino Gutiérrez en Cundinamarca, Antioquia y Nariño.

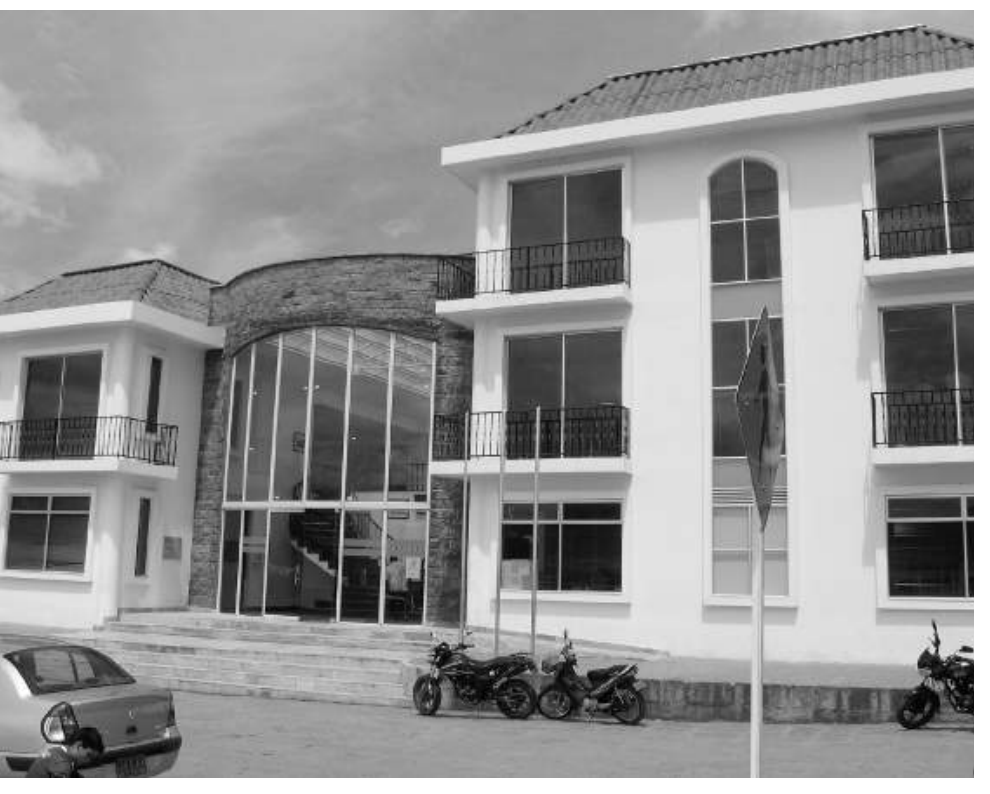

Figura 4:

Sistema constructivo típico de casas consistoriales en tierra

Fuente:

elaboración propia

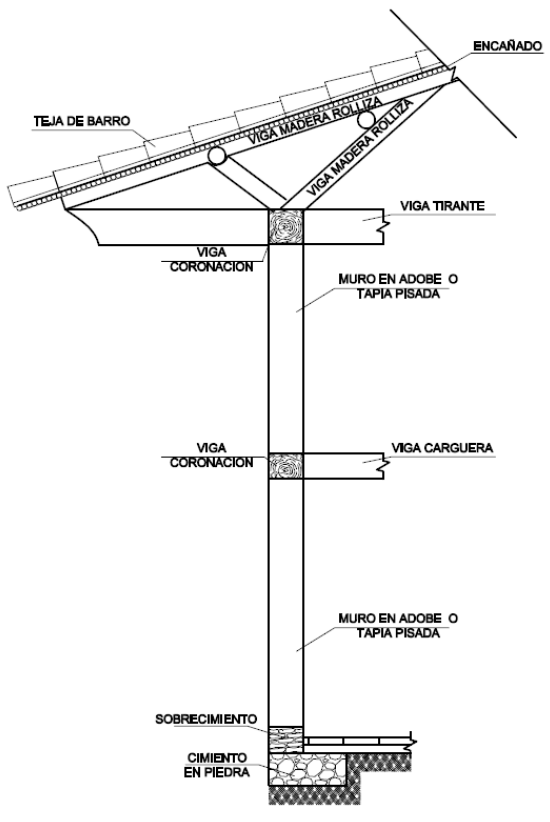

Las edificaciones se construyeron con una tipología sencilla que permitía que se desarrollaran allí las funciones establecidas por la corona española. Se localizaban en el marco de la plaza principal con forma de paralelogramo rectangular (figura 1a), cuya mayor longitud correspondía a la fachada principal del edificio. Poseían dos plantas en las cuales se distribuían diferentes actividades referentes al gobierno de la ciudad: despachos, salones del cabildo y la audiencia, escribanías, archivos, coso, cárceles (mujeres y hombres) y hospedaje de la guardia (Vigo, 1995). Gutiérrez (1921) menciona que allí también funcionaban escuelas públicas, usualmente las de niñas.

\section{Casas consistoriales en Cundinamarca}

En general las casas consistoriales de la época colonial en Colombia son edificaciones de dos pisos, con pilares de madera sobre los corredores de la fachada principal. Estas se caracterizan por tener simetría tanto en planta como en fachada, con algunas excepciones en las cuales el acceso al segundo piso se ubicaba en alguno de los costados del corredor (Curtido y Salgado 2006; Gutiérrez, 1990; Corradine, 1969) (figura 2).

Mencionan Gutiérrez (1921), Holton (1981), Suárez (2003), Rivas (1972) y Caballero (1974) que para 1886 existían en Cundinamarca 21 casas consistoriales con estas características (tabla 1). Adicionalmente a las mencionadas por estos autores, se encontraron siete edificaciones en otros municipios del departamento. Actualmente se conservan 11 de estas construcciones, que equivalen al 39\% del total.

Casas como las de Tocaima, Madrid, Fúquene, Carmen de Garupa, Funza y Mosquera sirven en la actualidad como instalaciones de la alcaldía.

En muchos lugares la casa consistorial fue demolida y en su lugar se construyeron edificaciones más modernas, las cuales ya no conservan la tipología de la época colonial pero aún reciben el nombre de casas consistoriales, como es el caso de Chipaque (figura 3). 


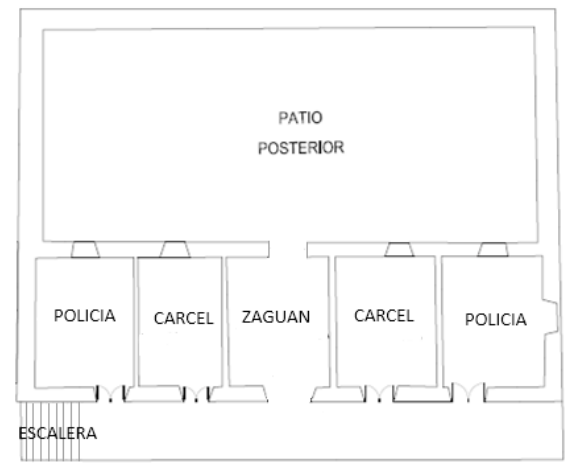

Las casas consistoriales de Cundinamarca que aún existen han sufrido modificaciones. Sin embargo, en ellas todavía se puede observar la simetría en fachada y los corredores con pilares de madera. En las casa de Simijaca y Sutatausa aún se conserva el acceso al segundo piso en uno de los costados del corredor.

En cuanto al sistema constructivo y los materiales, las casas consistoriales de la época colonial están conformadas por cimientos en piedra, pilares en madera, muros en adobe 0 tapia pisada, y entrepiso y cubierta en madera, esta última conformada por un sistema de par

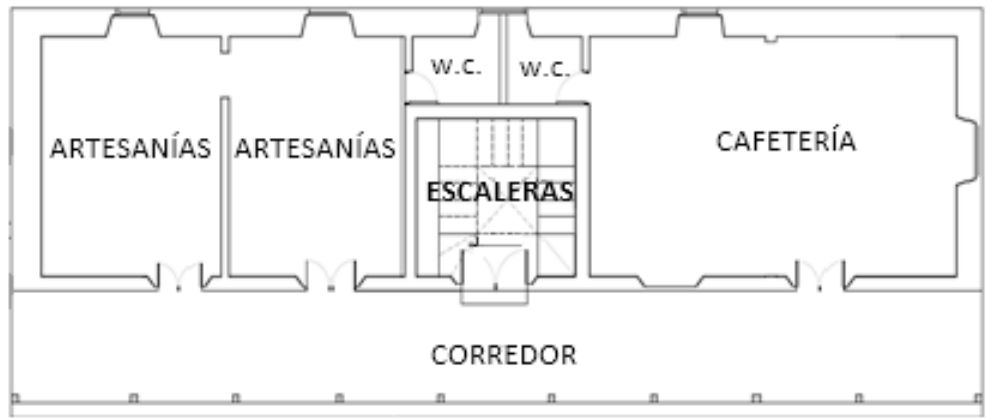

y nudillo con acabado en encañado, tierra y teja de barro (figura 4).

Rufino Gutiérrez (1921) destaca el mobiliario que poseían estas edificaciones: mesas, taburetes, escaños, estantes, baranda que dividía la oficina en dos partes. Para el caso de Engativá se relaciona la presencia de urnas, necesarias durante la época electoral, que habitualmente se empleaban como asientos.

En la actualidad este tipo de edificaciones no están siendo valoradas. En la mayoría de los casos los pobladores desconocen el carácter histórico de la edificación, razón por la cual las
Figura 5:

Distribución esquemática

de los espacios del

primer piso de lacasa

Cogua en sus inicios

Fuente:

elaboración propia

Figura 6:

Distribución esquemática de los espacios de

la Casa Cogua en la actualidad. a) Primer piso, b) Segundo piso.

Fuente:

elaboración propia
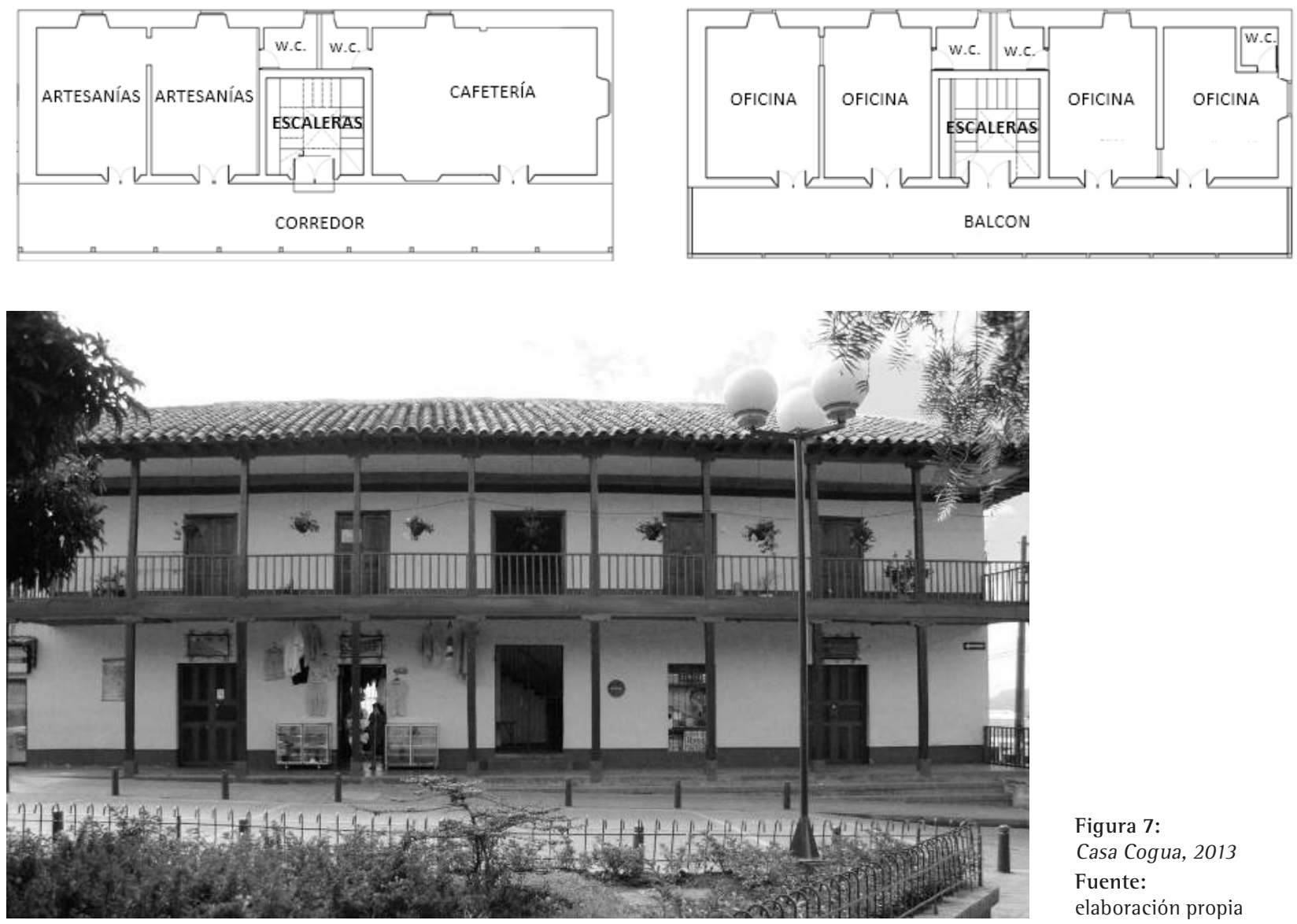

Figura 7:

Casa Cogua, 2013

Fuente:

elaboración propia 
Figura 8:

Planta arquitectónica del primer piso de la casa consistorial de Cogua

Fuente: elaboración propia

Figura 9:

Planta arquitectónica del segundo piso de la casa consistorial de Cogua

Fuente: elaboración propia

Figura 10: Fachada principal casa consistorial de Cogua

Fuente: elaboración propia
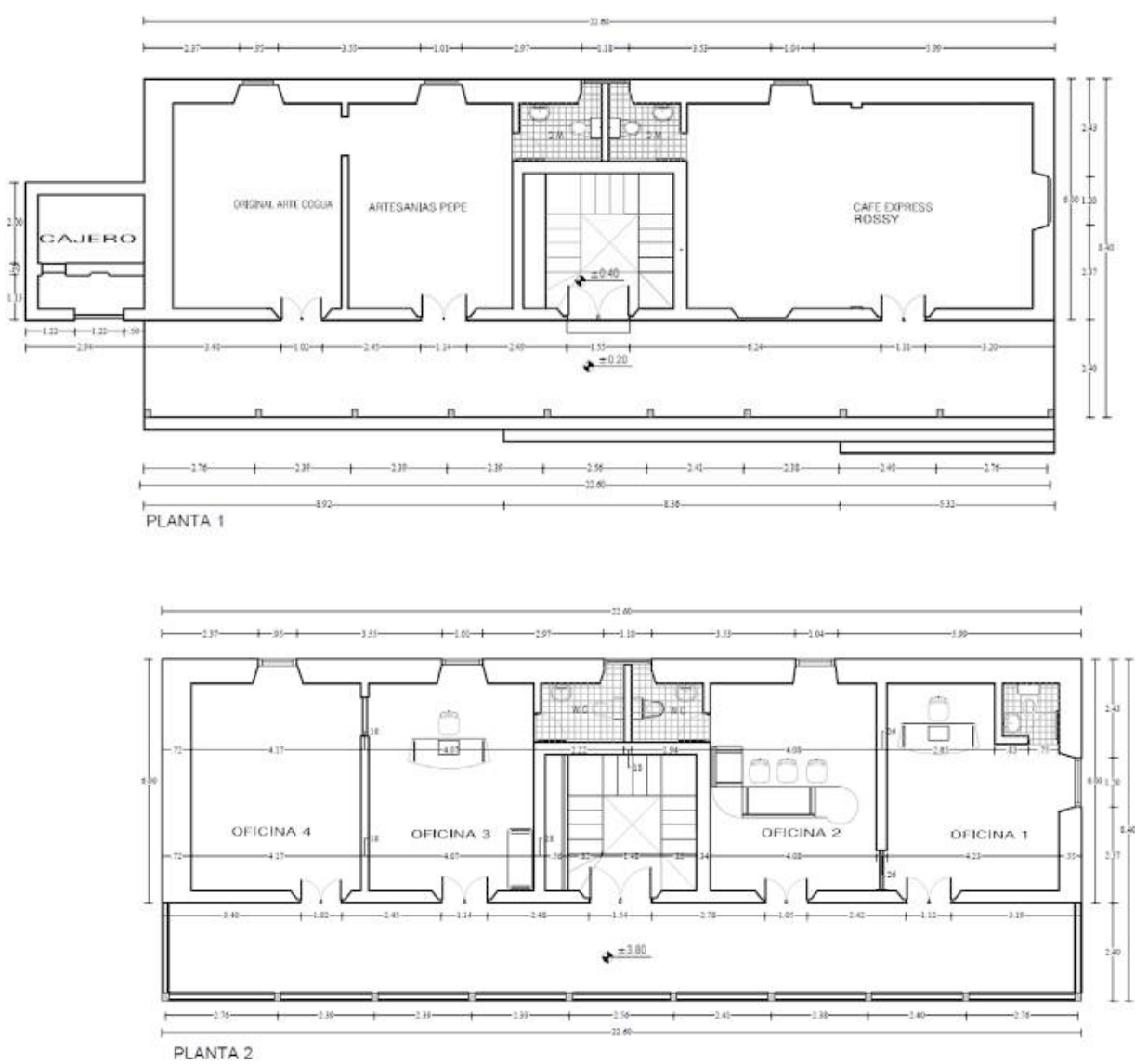

PLANTA 2

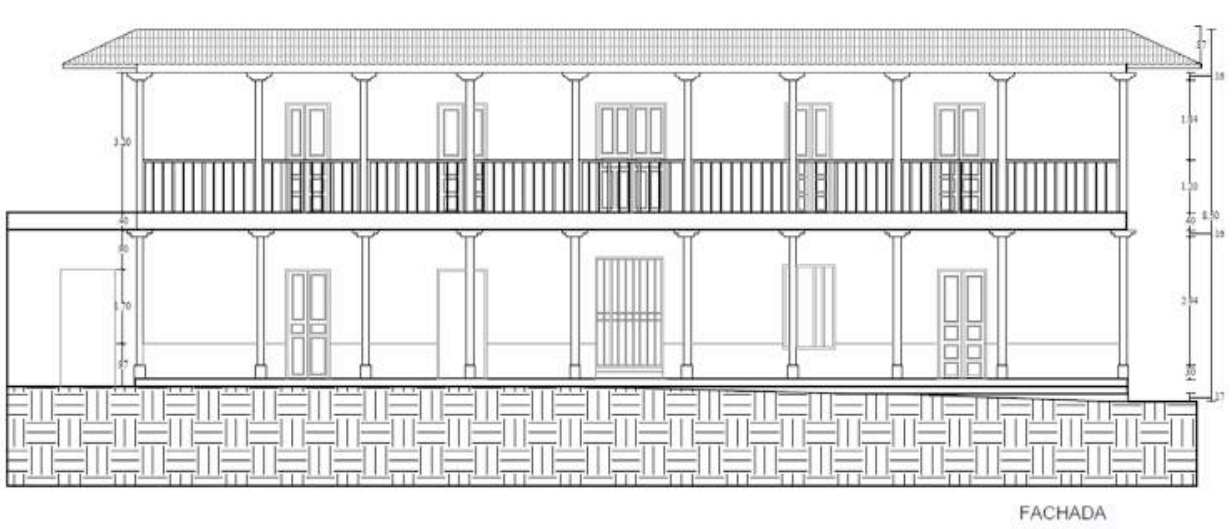

Figura 11: Fachada lateral casa consistorial de Cogua

Fuente: elaboración propia

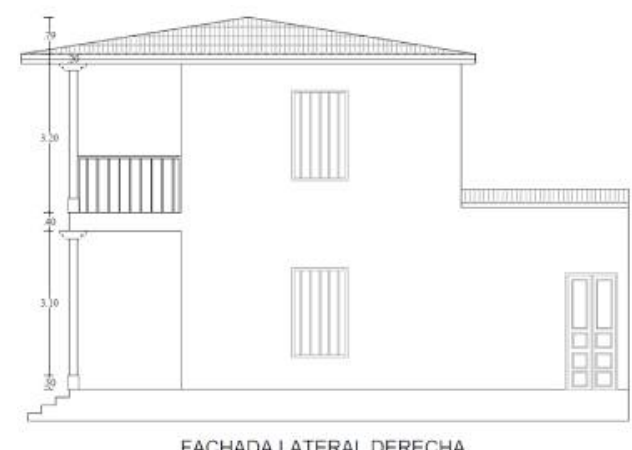

FACHADA LATERAL DERECHA casas consistoriales sufren reformas sin respetar su tipología, y en ocasiones son demolidas para dar paso a edificaciones modernas.

Caso de estudio: casa consistorial de Cogua

Cogua es un municipio de Cundinamarca ubicado aproximadamente a $50 \mathrm{~km}$ de la ciudad de Bogotá, por salida norte. Sobre uno de los costados de la plaza central del pueblo se encuentra, en 

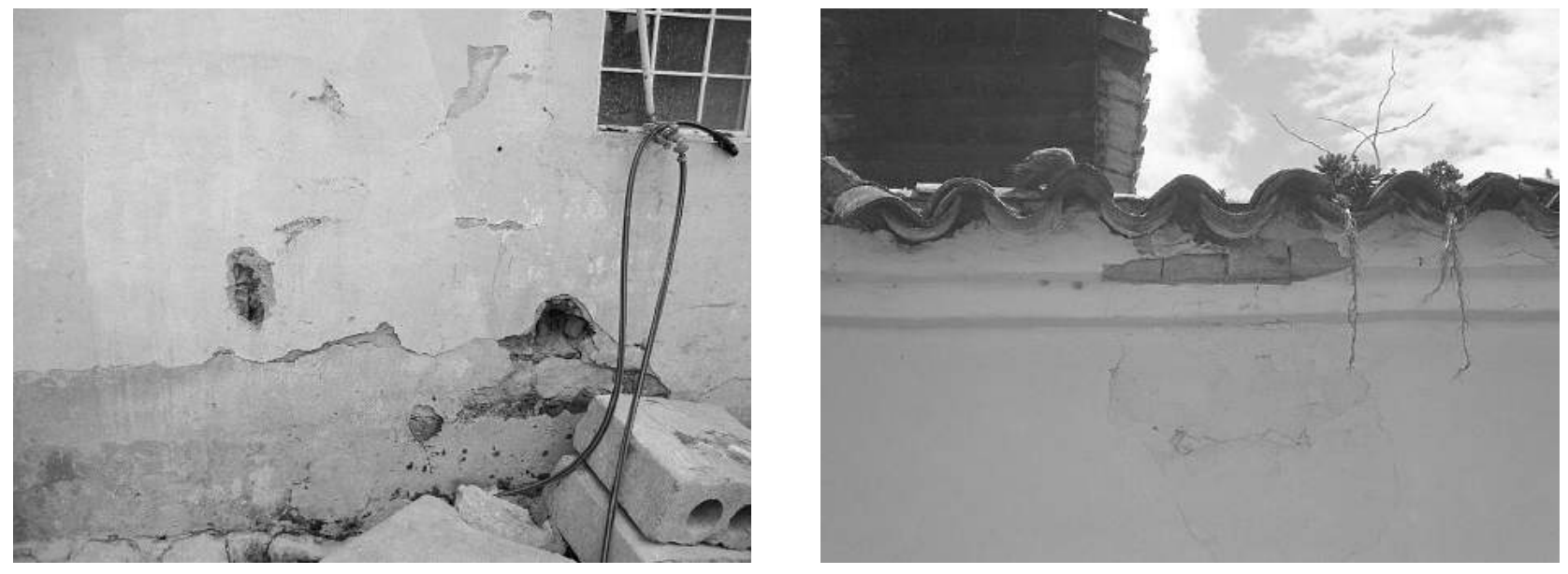

una esquina, la casa consistorial (figura 1b). No existe evidencia de cuándo fue construida. Sin embargo, se cree que fue fundada entre 1780 y 1800 (Curtido y Salgado, 2006).

La casa consistorial de Cogua es una edificación en dos pisos que ha sufrido modificaciones a lo largo del tiempo. Originalmente la casa tenía el acceso al segundo piso por el costado derecho del corredor, contaba con un patio posterior, casi igual de grande al espacio que ocupaba la casa, al cual se accedía por medio de un zaguán en el primer piso (Curtido y Salgado, 2006) (figura 5). En la actualidad la edificación ya no cuenta con patio, las escaleras fueron eliminadas del costado y se encuentran ubicadas sobre el sector que era antiguamente el zaguán (figura 6). Sin embargo, la casa consistorial de Cogua aún conserva características propias de las casas consistoriales de la época colonial. En ella se aprecian sus pilares en madera sobre corredor y balcón, así como su simetría en fachada (figura 7).

El uso de la edificación también ha cambiado con el tiempo. Inicialmente el primer piso estaba destinado para la cárcel y en el segundo

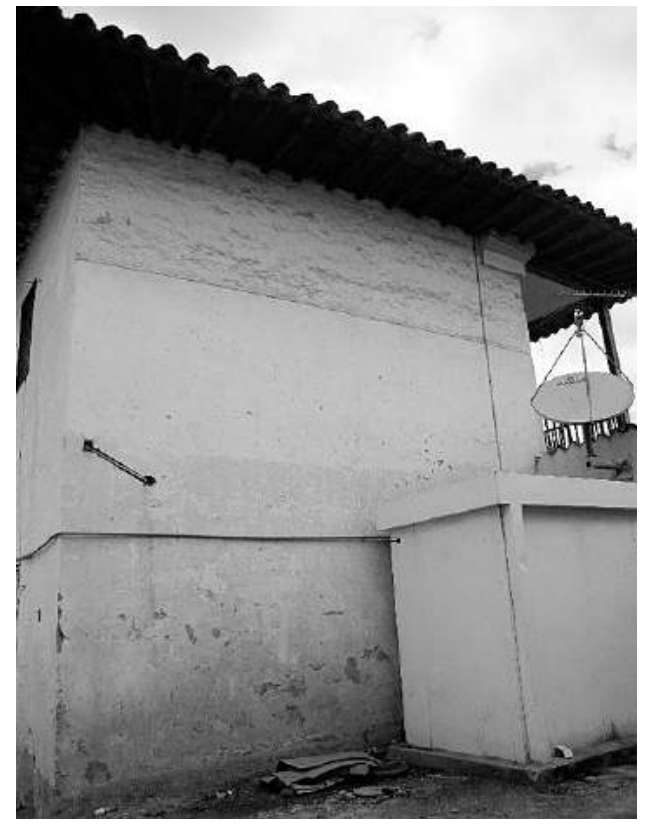

piso se ubicaban las oficinas para la administración y representación legal del pueblo. Según investigación realizada por Curtido y Salgado (2006), en 1946 la edificación fue usada como escuela municipal. En 1965 se usó el primer piso para estación de policía y el segundo para centro
Figura 12:

Patologías en muros de adobe de la Casa Cogua: a) Erosión del adobe, b) Presencia de microorganismos, c) Humedad y suciedad

Fuente:

elaboración propia

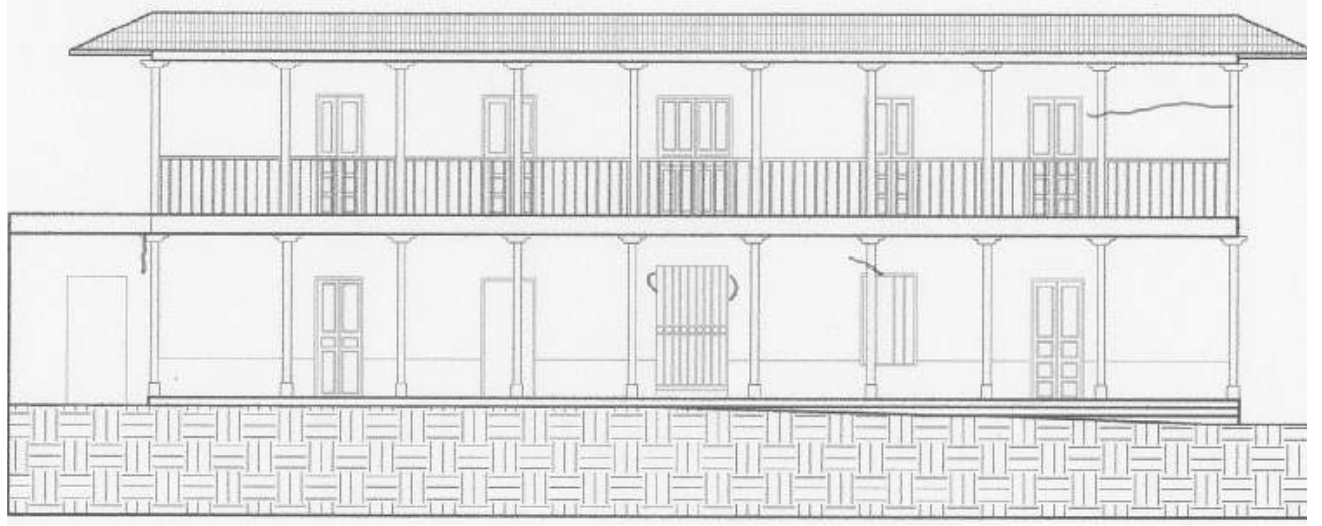

Figura 13:

Fisuras en muros de fachada principal de la casa consistorial de Cogua

Fuente:

elaboración propia 


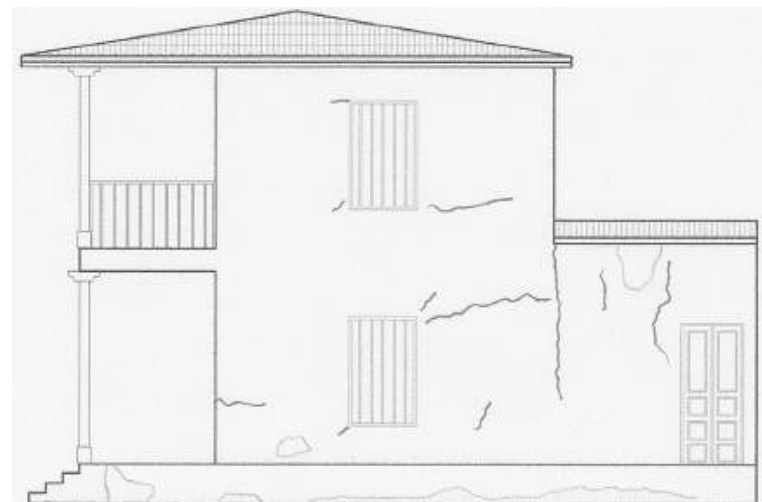

Figura 14: Fisuras en muros de fachada lateral de la casa consistorial de Cogua

Fuente: elaboración propia muros exteriores (figura 12). Algunos muros pre- de salud. A partir de 1978, se destina para a uso comercial y el segundo nivel se usa para oficinas de carácter municipal.

En las figuras 8, 9, 10 y 11 se presentan la distribución arquitectónica actual y el uso de los espacios de la casa Cogua.

A continuación se describen brevemente los elementos constructivos de la casa Cogua, así como las patologías encontradas.

\section{Muros}

Construidos en adobe con secciones de 80,70 y $60 \mathrm{~cm}$ de espesor que sirven de sistema estructural a la edificación. Algunos muros presentan humedades, erosión, microorganismos y suciedad debido a la falta de protección y mantenimiento; estas patologías se observan principalmente en sentan fisuras por cortante en vanos de puertas $\mathrm{y}$ ventanas (figura 13).

Debido a las modificaciones que ha sufrido la casa fue necesaria la construcción de nuevos muros, los cuales fueron levantados con bloques de arcilla cocida, y se ubican en el sector de las escaleras y en cerramientos de instalaciones sanitarias.
Los muros en adobe cuentan con viga de coronación en madera, tanto a nivel de entrepiso como de cubierta.

\section{Columnas}

Son elementos cuadrados de $15 \mathrm{~cm}$ ubicados en la fachada principal de la casa en las galerías en los pisos primero y segundo. En el primer nivel están apoyados sobre pedestales de $30 \mathrm{~cm}$ de alto y sección cuadrada de $20 \mathrm{~cm}$.

Algunas columnas presentan daños en la base por descomposición de la madera (figura 16), esto ocasiona pérdida de resistencia del elemento debido al cambio de densidad, de sección y de área de apoyo.

Otras patologías observadas en los pilares de madera es el agrietamiento, desplazamiento de la base y pérdida de verticalidad del elemento (figura 16).

\section{Entrepiso}

Está conformado por vigas en madera con sección aproximada de $10 \times 15 \mathrm{~cm}$, sobre las cuales se apoya transversalmente un entramado en madera que da soporte al acabado de piso en el mismo material.

Se encontró que el piso del balcón fue remplazado por baldosas sobre una placa de concreto reforzado de aproximadamente $10 \mathrm{~cm}$ de espesor (figura 17). En las vigas del entrepiso del sector del balcón se observa daño de la madera (figura 17), y el cambio en su densidad es notorio al tacto.

\section{Escalera}

Inicialmente estaba ubicada en el costado derecho del corredor y, según Curtido y Salgado (2006)

Figura 15:

Fisuras en muros de fachada posterior de la casa consistorial de Cogua Fuente: elaboración propia

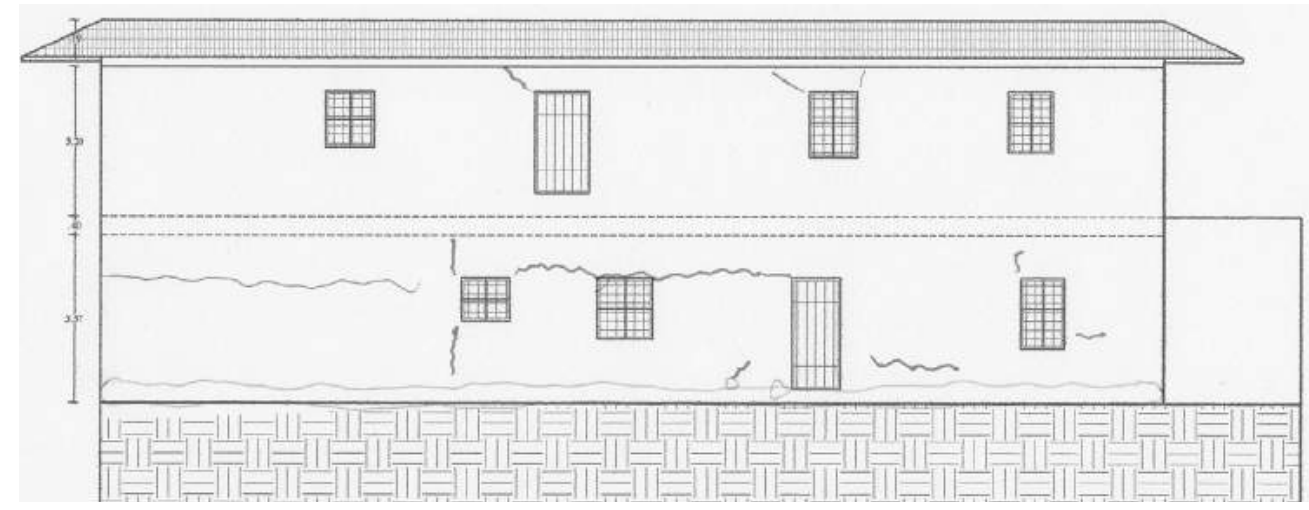



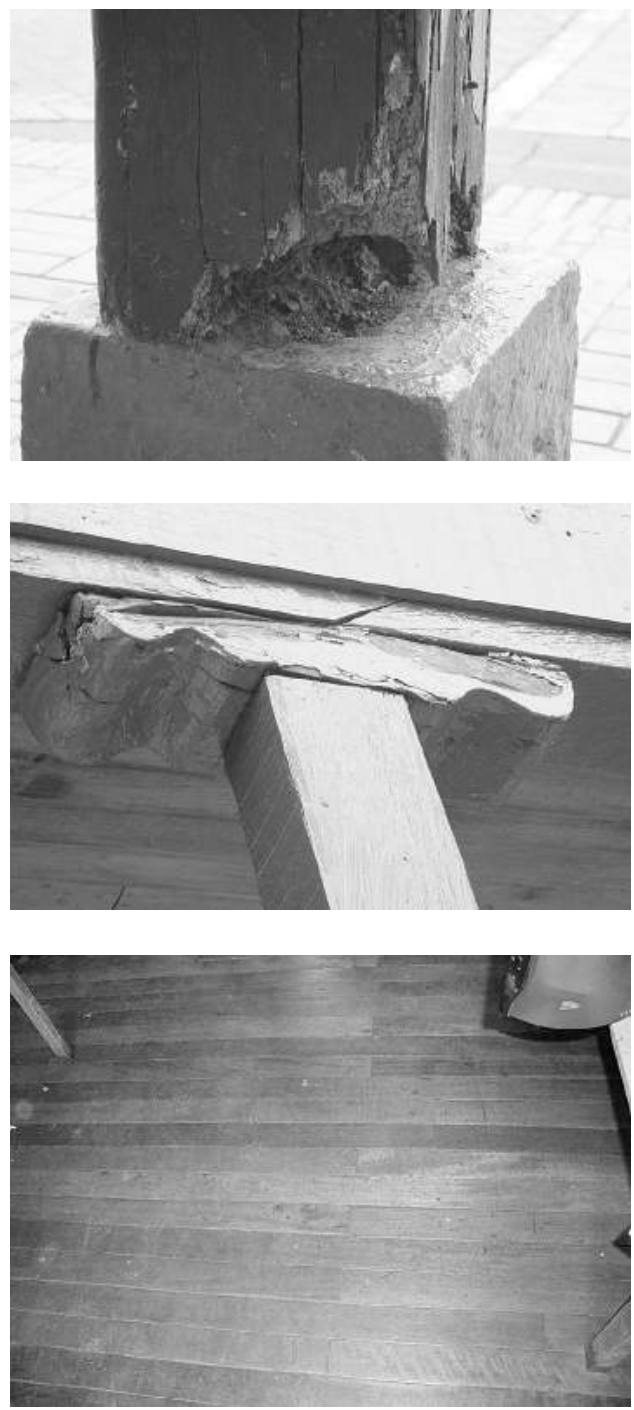

era de madera, pero por su deterioro tuvo que ser demolida. La actual fue construida en concreto y está ubicada en el espacio del antiguo zaguán (figura 18).

\section{Cielo raso}

Se encuentra suspendido de las vigas de entrepiso y cubierta. Está conformado por chusque, tierra y pañetado en cal (figura 19).

Para disminuir el peso de la edificación, el cielo raso del segundo piso fue demolido, exceptuando el ubicado sobre el sector de escaleras, el cual aún se conserva por tener pintura mural (figura 20), aunque presenta múltiples fisuras.

El cielo raso sobre el sector del corredor presenta deformaciones y fisuras, debidas muy seguramente a su gran peso (figuras 21 y 22).

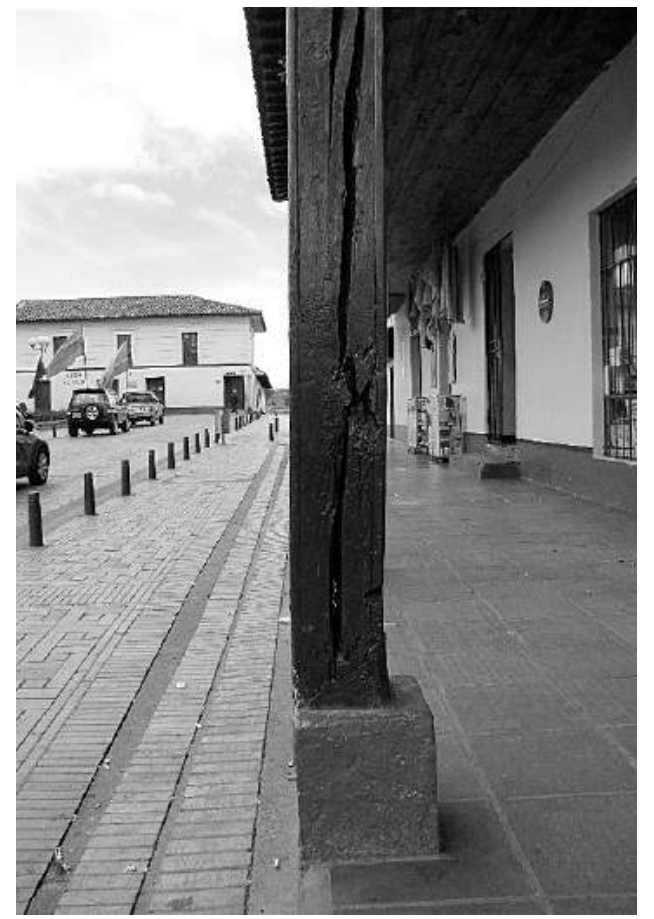

Figura 16:

Patologías en los pilares de madera: a) Pérdida de sección por descomposición de la madera, b) Agrietamientos, desplazamiento y pérdida de verticalidad de los pilares en madera, c) Aplastamiento de los capiteles de las columnas Fuente:

elaboración propia
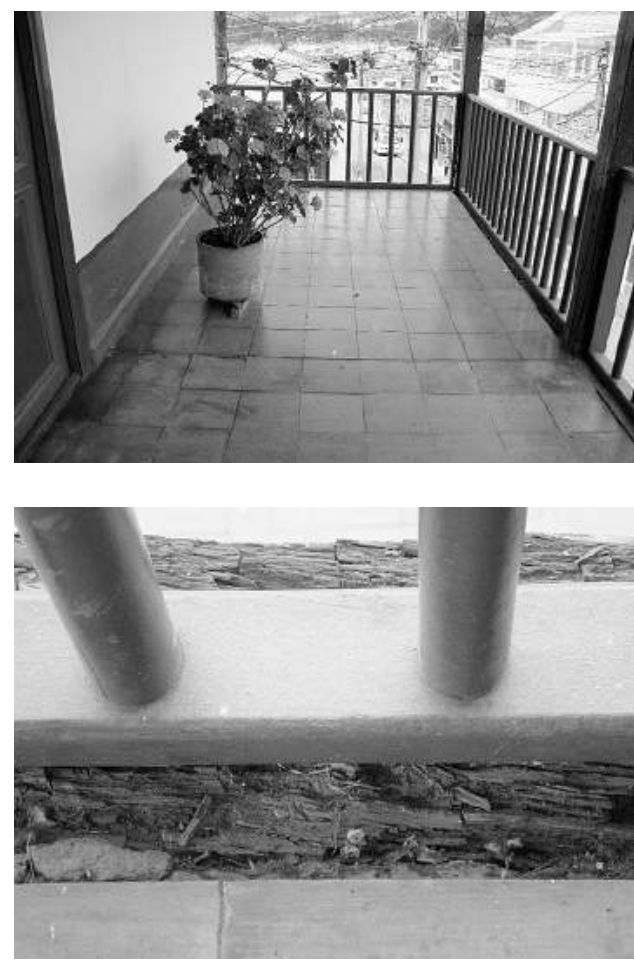

Figura 17:

Entrepiso de la casa Cogua: a) Piso en madera, correspondiente al sector de oficinas, $b$ ) Piso en baldosa sobre placa de concreto reforzado, sector del corredor de segundo piso de la casa, c) Vigas de entrepiso deterioradas Fuente: elaboración propia

\section{Cubierta}

La estructura está conformada por elementos de madera y su tipología corresponde a cercha de par y nudillo (figura 23). Los amarres son con fibras y las uniones en caja. El acabado de cubierta es en encañado, tierra y teja de barro.

La cubierta presenta grandes deformaciones que están causando problemas de goteras 


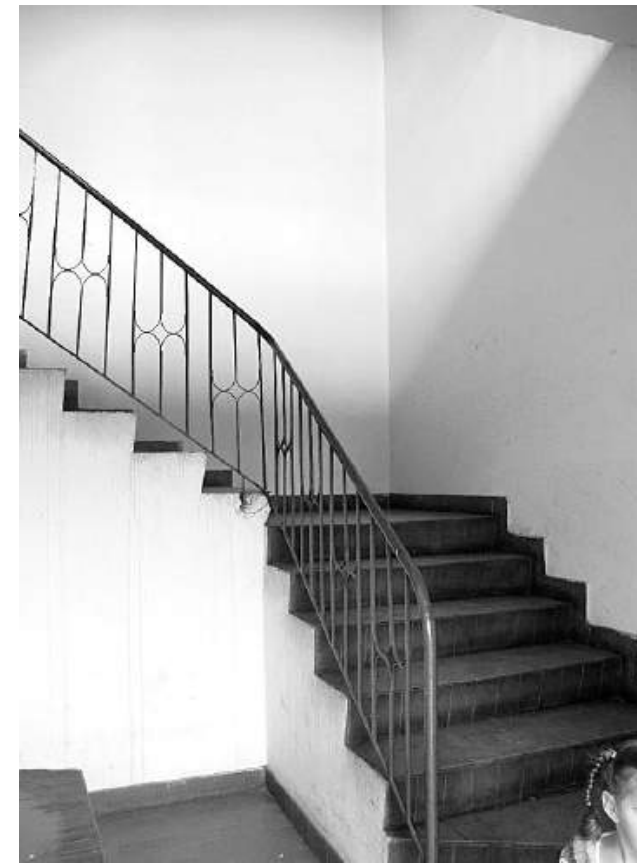

elaboración propia

ista act

s escaleras.

Fuente:

Figura 20: Pintura mural sobre el cielo raso del sector de las actuales escaleras.

Fuente: Archivo de los autores

Figura 19: Cielo raso suspendido de las vigas de cubierta.

Fuente: elaboración propia
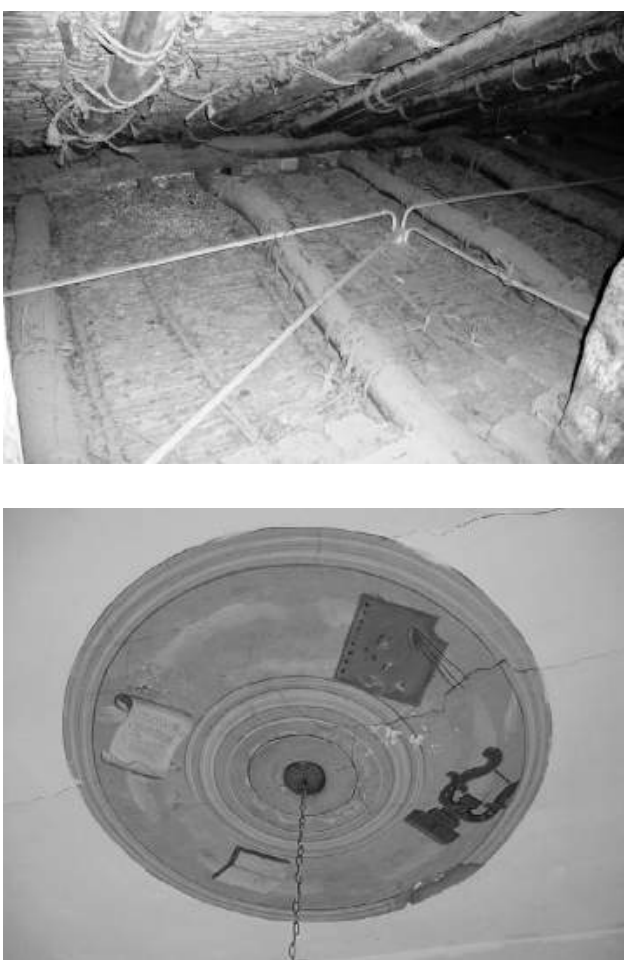

Figura 21: Cielos rasos sobre el sector del corredor del segundo piso, que presenta deformaciones $y$ fisuras Fuente: Archivo de los autores

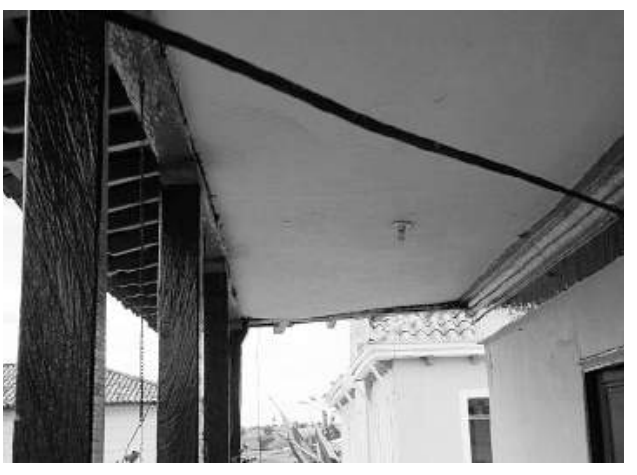

dentro de la edificación. Las maderas de los aleros de cubierta presentan descomposición en algunos sectores debido a la humedad (figura 24).

\section{Puertas y ventanas}

La mayoría de estos elementos son originales de la edificación y se encuentran en buen estado (figura 25). La puerta de acceso a las escaleras, antiguo zaguán, fue cambiada por una puerta en reja metálica y una de las puertas de la fachada principal fue cambiada por un ventanal en reja.

En la actualidad la casa consistorial se encuentra en relativo buen estado. Sin embargo, es sabida la carencia de resistencia sísmica de los sistemas constructivos en tierra, debido a la poca resistencia a la flexión en su plano (Yamín, et al., 2007; Meli et al., 1980; Navarro et al., 2009; Blondet et al., 2003; Blondet et al., 2008). Esto hace necesario llevar a cabo un análisis numérico de la resistencia de la edificación ante posibles movimientos sísmicos. Es válido recordar que el que una edificación haya permanecido en pie durante mucho tiempo, no es garantía de que sea sismorresistente. $\mathrm{Al}$ respecto, es conocido el caso de la ciudad de Arg-é Bam, Irán, la cual estaba urbanizada con edificaciones en adobe que habían permanecido en pie desde el año 500 a. C., y que en 2003 fue destruida por un movimiento sísmico (UNESCO, 2003).

\section{Modelo numérico}

Para el análisis numérico de la edificación se usó el programa SAP 2000. El análisis sísmico se realizó bajo los parámetros que contempla la NSR-10 para edificaciones existentes.

El modelo utilizado es una aproximación numérica, ya que no existe un estudio detallado del comportamiento de las propiedades mecánicas del material de la edificación, ni existe en la actualidad un software de elementos finitos que tenga en cuenta un modelo constitutivo para el adobe. Lo que se pretende mostrar con la modelación numérica son los puntos críticos de la edificación y cómo pueden reducirse los esfuerzos a los que se encuentran sometidos los muros mediante la aplicación de una técnica de reforzamiento para edificaciones en tierra.

Para modelar los muros de la edificación, tal como se encuentra en su estado actual, se usaron elementos tipo Shell discretizados cada 

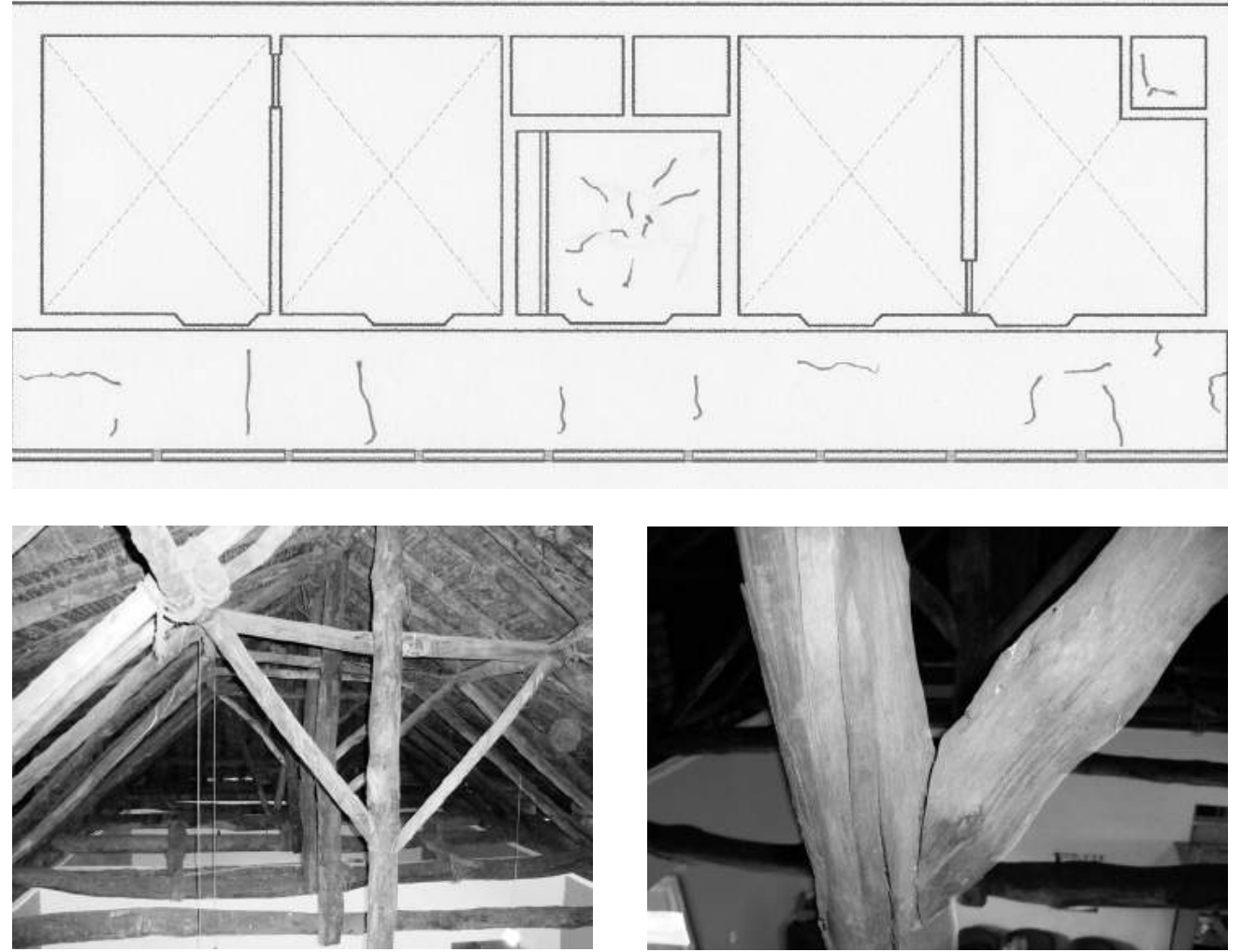

$20 \mathrm{~cm}$. Para la modelación de los pilares de madera y las vigas, de entrepiso y cubierta, se usaron elementos tipo Frame (figura 26). El análisis fue realizado para cargas gravitacionales (peso propio, cargas muertas sobreimpuestas y cargas vivas de uso oficinas) y para evento sísmico, con probabilidad del veinte por ciento de ser excedidos en cincuenta años (figura 27). Los parámetros símicos y las cargas sobreimpuestas a la estructura se relacionan en la tabla 2 .

En el análisis numérico se obtuvieron desplazamientos a nivel de cubierta de $17,58 \mathrm{~cm}$, correspondientes a derivas del orden del 2,38\%. Aunque este tipo de estructura no puede ser clasificado dentro de ninguno de los sistemas estructurales que contempla la NSR-10, vale la pena recordar que para edificios en mampostería la deriva máxima que permite dicho reglamento es de $0,5 \%$.

Para cargas gravitacionales, la edificación no presenta grades esfuerzos sobre sus elementos. Sin embargo, al incluir las fuerzas sísmicas se observan grandes concentraciones de esfuerzos, principalmente en esquinas de muros y en vanos de puertas y ventanas (figura 28).

De acuerdo con Ruiz et al. (2012), la resistencia máxima a la tensión de las edificaciones de adobe es de $20 \mathrm{kPa}$. Según los resultados ob-
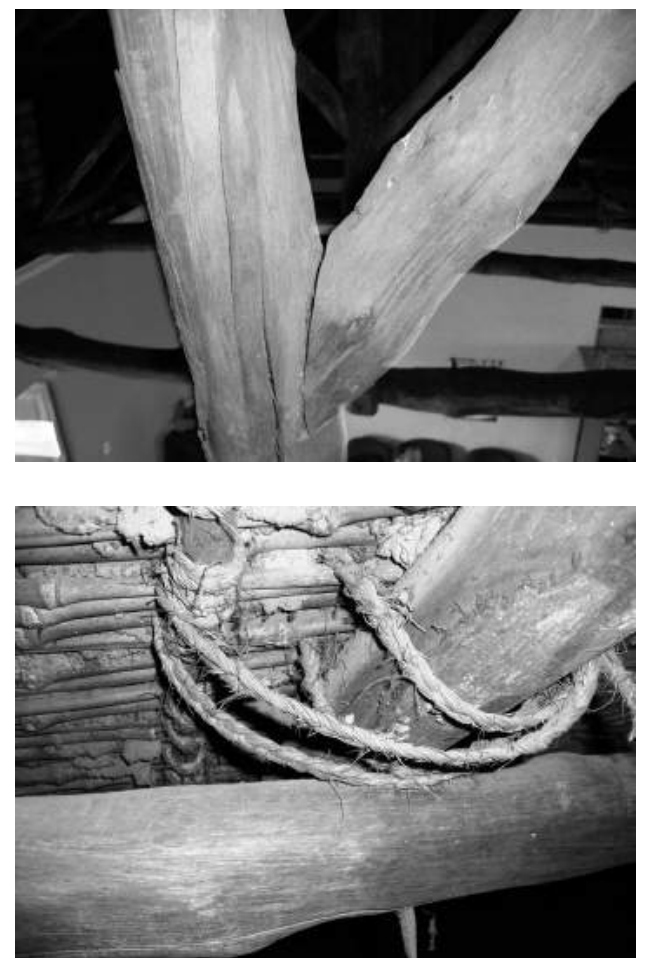

Figura 22:

Fisuras en el cielo raso del segundo pisos sobre el sector del balcón y escaleras. Fuente: elaboración propia

Figura 23:

a) Distribución de los elementos de cubiertas, b) Uniones tipo caja en elementos de cubierta, c) Uniones con fibras vegetales en elementos de cubierta. Fuente:

elaboración propia

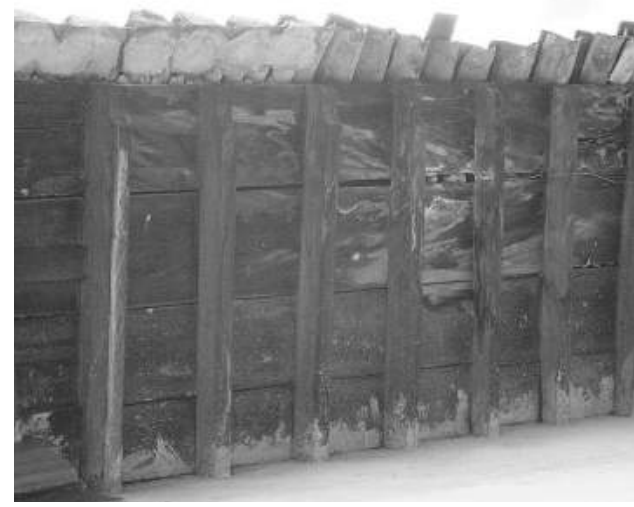

Figura 24:

Descomposición de la madera en elementos de cubierta.

Fuente:

Archivo de los autores

tenidos en la modelación numérica, los esfuerzos máximos a tensión de los muros de adobe de la casa consistorial de Cogua son de $240 \mathrm{kPa}$. De acuerdo con esto, se pudo determinar que la edi- 
Tabla 2: Parámetros sísmicos y cargas utilizadas para la modelación numérica

\begin{tabular}{|c|c|c|}
\hline \multicolumn{2}{|c|}{ Zona de amenaza sísmica } & Intermedia \\
\hline \multicolumn{2}{|c|}{ Coeficiente de aceleración pico efectiva (Aa) } & 0.15 \\
\hline \multicolumn{2}{|c|}{ Coeficiente de aceleración piso efectiva reducido (Ae) } & 0.09 \\
\hline \multicolumn{2}{|c|}{ Coeficiente de aceleración de velocidad horizontal pico efectiva (Av) } & 0.2 \\
\hline \multicolumn{2}{|c|}{ Coeficiente de capacidad de disipación de energía $(\mathrm{R})$} & 1.0 \\
\hline \multicolumn{2}{|l|}{ Coeficiente de importancia (I) } & 1.0 \\
\hline \multirow{3}{*}{ Cargas vivas $\left(\mathrm{kN} / \mathrm{m}^{2}\right)$} & Entrepisos & 2.0 \\
\hline & Corredores y escaleras & 3.0 \\
\hline & Cubierta & 0.35 \\
\hline \multirow{2}{*}{ Carga muerta sobreimpuesta $\left(\mathrm{kN} / \mathrm{m}^{2}\right)$} & Entrepisos* & 2.0 y 4.0 \\
\hline & Cubierta & 3.1 \\
\hline \multirow{3}{*}{\multicolumn{2}{|c|}{ Combinaciones de carga usadas en el análisis }} & $1.2 \mathrm{D}$ \\
\hline & & $1.2 \mathrm{D}+1.0 \mathrm{~L}$ \\
\hline & & $1.2 \mathrm{D}+1.0 \mathrm{~L}+1.0 \mathrm{E}$ \\
\hline
\end{tabular}

* Para el entrepiso se tiene una carga para el sector de oficinas diferente a la del corredor. Fuente: elaboración propia

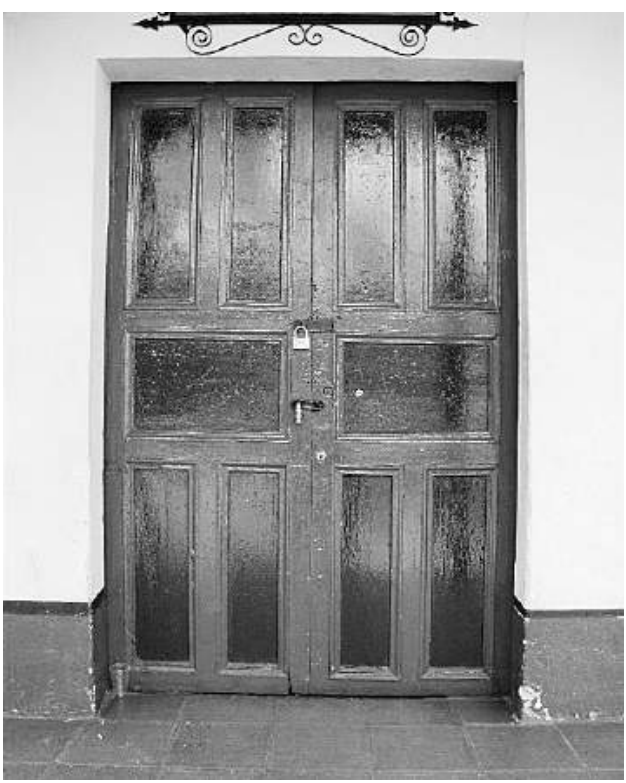

Figura 25: a) Puerta en fachada principal (Café Rossi), b) Puerta fachada lateral de la casa, c) Ventana fachada lateral, d) Ventana fachada posterior.

Fuente: elaboración propia
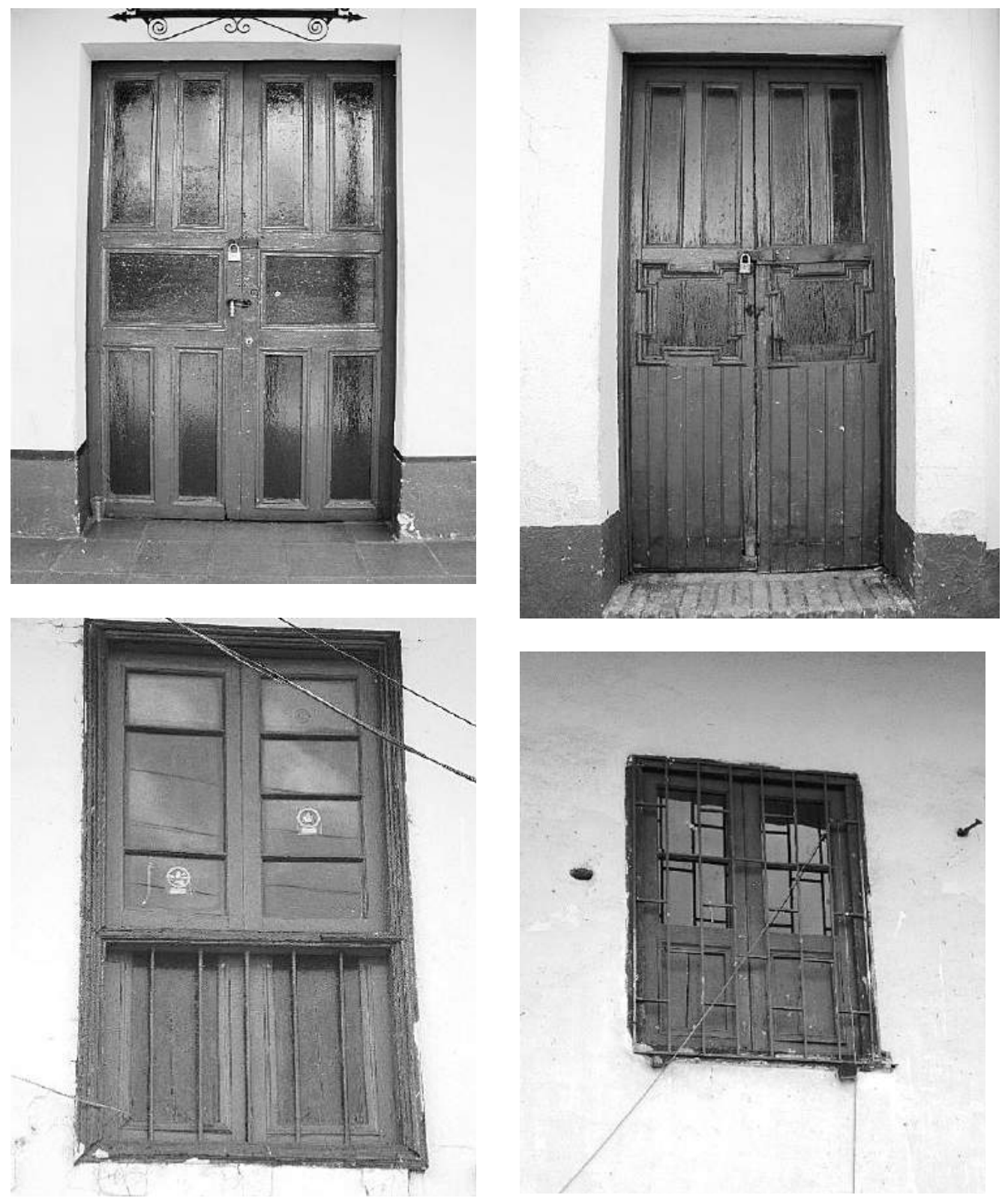
ficación no se encuentra en capacidad de soportar el sismo de diseño que contempla la nsr-10, lo cual hace necesaria la implantación de una técnica de rehabilitación que garantice la preservación del inmueble.

Entre las técnicas que se han estudiado para la rehabilitación sísmica de edificaciones en tierra se encuentran principalmente tres (López et al., 2007; Blondet et al., 2008; Yamín, et al., 2007; Blondet et al., 2003; Yamín, 2003; Navarro et al., 2009; AlS, 2005):

- Reforzamiento sísmico con mallas.

- Maderas confinantes.

- Tensores de acero (tierra postensada).

Para el estudio de la casa Cogua se optó por la técnica de rehabilitación con maderas confinantes. Esta técnica consiste en la colocación de
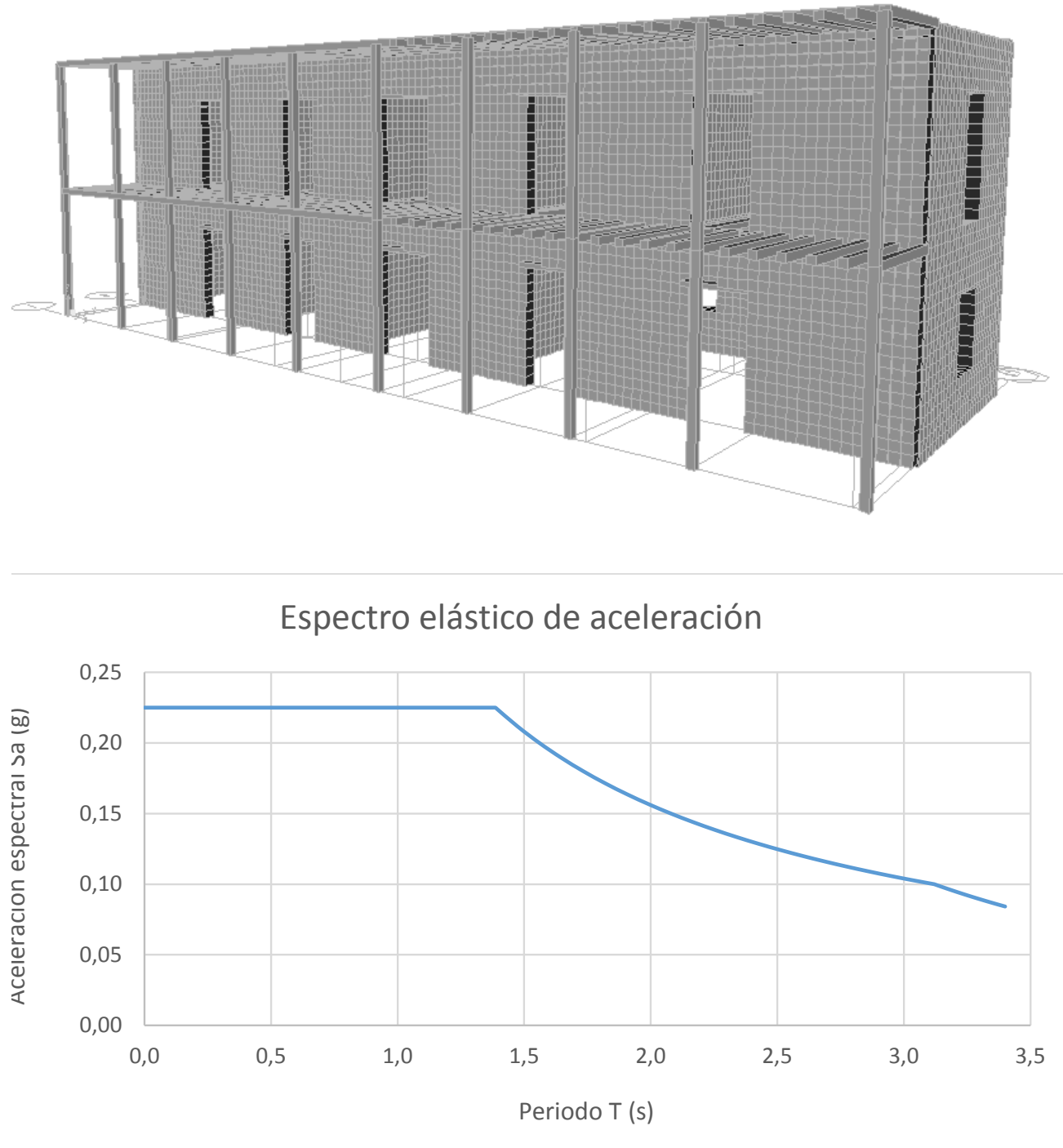

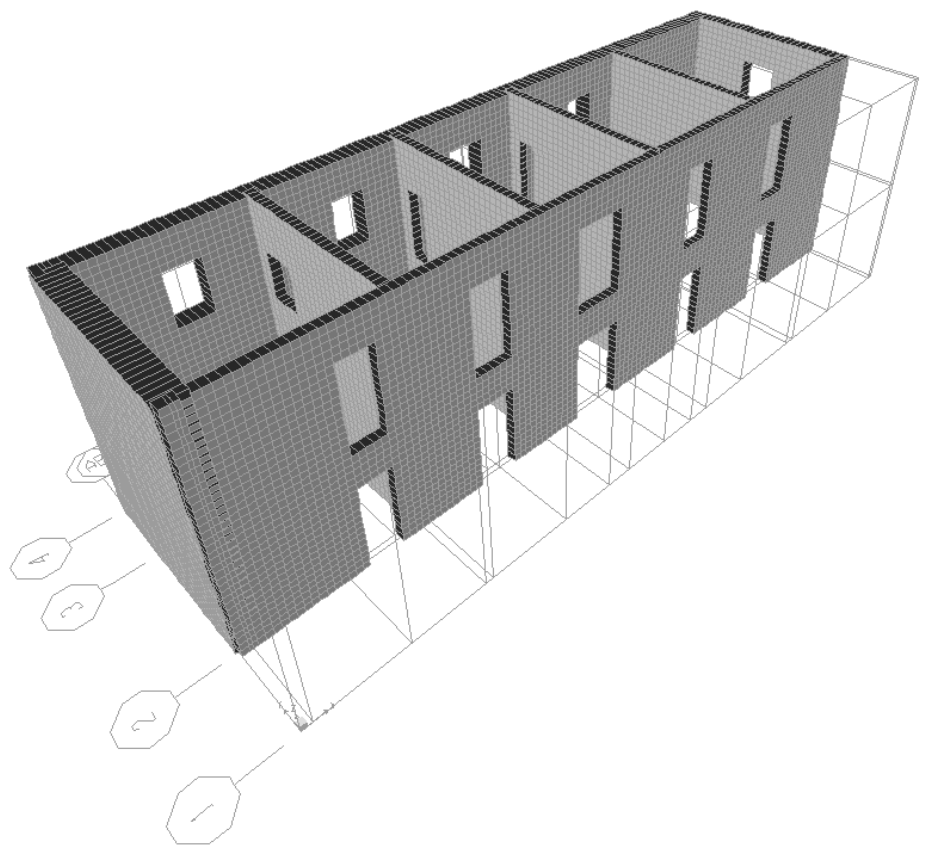

Figura 26:

Modelo en SAP 2000 de la casa consistorial de Cogua en su estado actual: a) Modelo con distribución de los muros, b) Modelo incluyendo muros, columnas en madera y vigas de entrepiso y cubierta. Fuente: elaboración propia

Figura 27:

Espectro elástico de aceleración con seguridad limitada, para el municipio de Cogua. Fuente: elaboración propia 
Figura 28: Esfuerzos en los muros ("102 kPa) de la Casa Cogua por cargas gravitacionales y sísmicas, en su estado actual. Fuente: elaboración propia

Figura 29: Imagen explicativa de la distribución de las maderas en el reforzamiento de muros en tierra. Fuente: elaboración propia

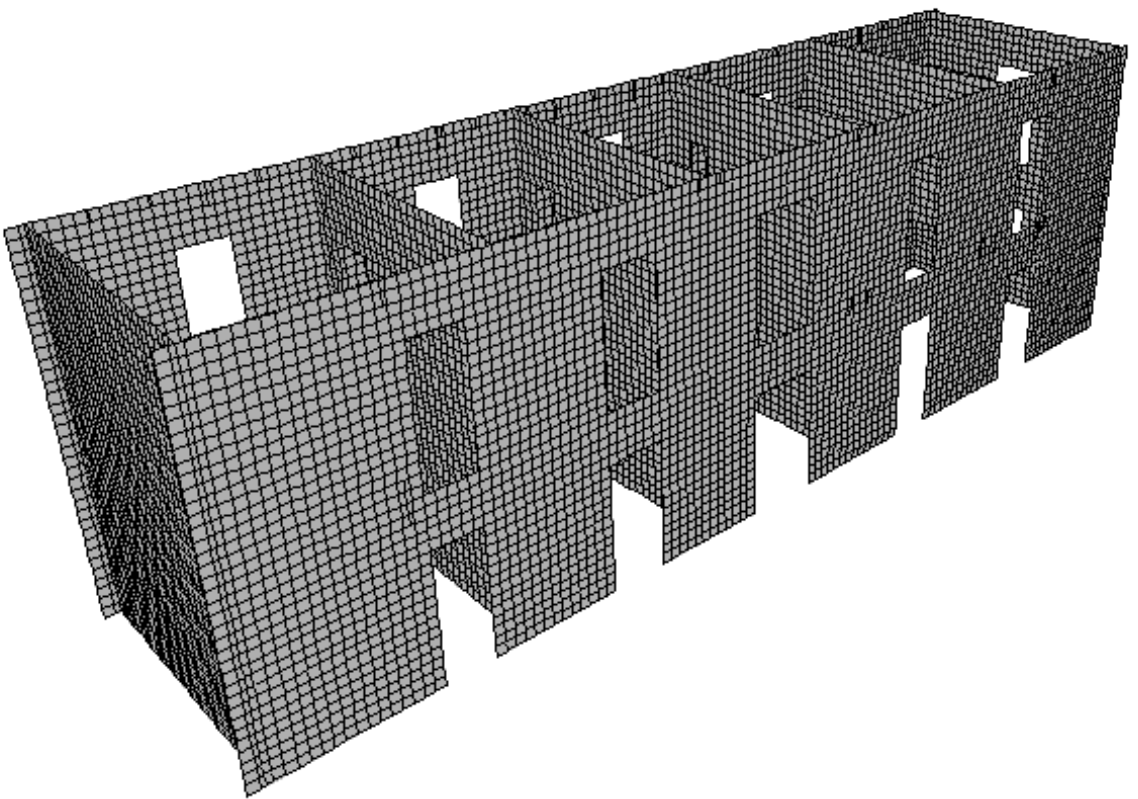

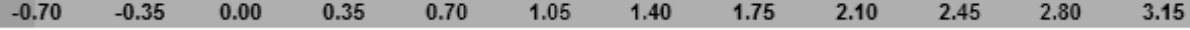

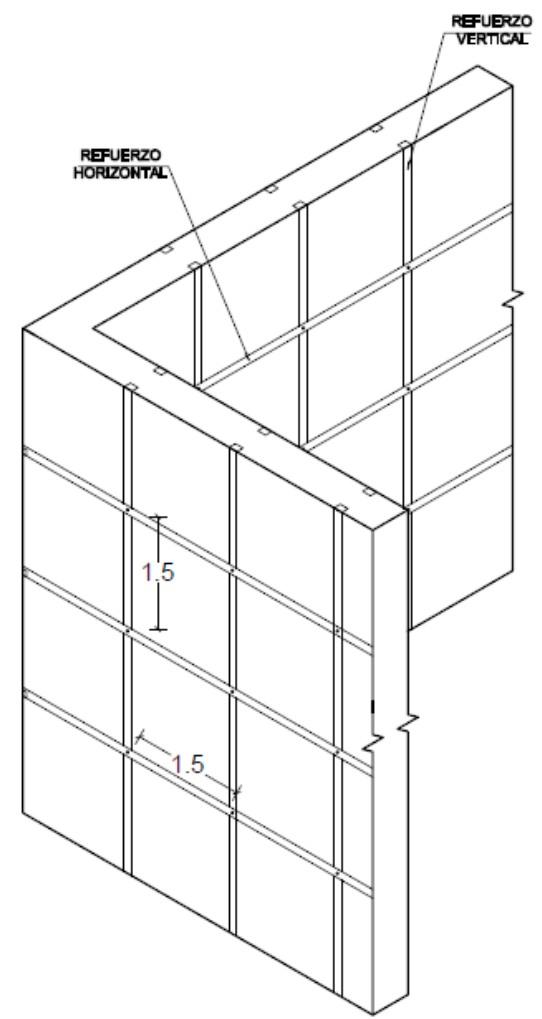

tablas de madera en sentido vertical y horizontal, tanto en la cara interna como externa del muro (figura 29), las cuales deben ir conectadas a través del muro por medio de pasadores de alambre 0 barra (AIS, 2005).

La modelación numérica se hizo con elementos tipo Solid, los cuales estaban ex- truidos cada $10 \mathrm{~cm}$. Para el reforzamiento se generaron elementos tipo Frame de $15 \mathrm{~cm}$ de ancho y $2 \mathrm{~cm}$ de espesor. Estos elementos fueron puestos en los muros con una separación no mayor a 1,50 m (figura 30). Las consideraciones de cargas gravitacionales y acciones sísmicas fueron iguales a las del modelo inicial (sin reforzar).

Entre los resultados obtenidos se aprecia la disminución en los desplazamientos, los cuales pasaron de 17,58 cm, en el modelo sin refuerzo, a $4,07 \mathrm{~cm}$ en el modelo reforzado con maderas confinantes. Esto representa una disminución en deriva del orden del 76\%.

En cuanto a esfuerzos, se obtuvieron valores máximos de $17 \mathrm{kPa}$ (figura 31), lo cual representa una disminución del 93\% respecto al modelo sin refuerzo.

La reducción significativa en los esfuerzos máximos a tensión y en las derivas de la edificación, hacen de la técnica de rehabilitación con maderas confinantes una alternativa viable para la conservación y preservación de la casa consistorial de Cogua.

Aunque la técnica de rehabilitación de maderas confinantes es invasiva, esta es adaptable fácilmente a la casa consistorial de Cogua, ya que su trazado y ubicación permite que los elementos de madera se coloquen en ambas caras de los muros. Adicionalmente, la casa no cuenta con ningún tipo de pintura que deba conservarse, lo 


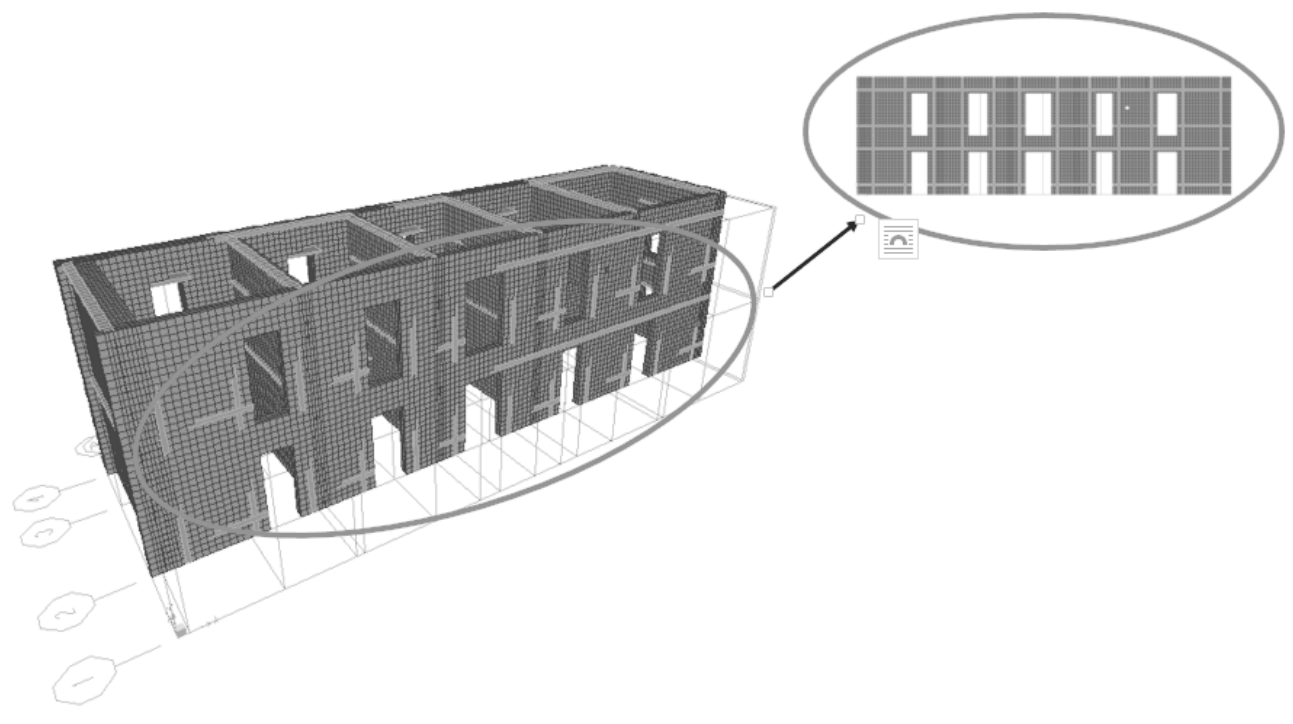

Figura 30:

Modelo en SAP 2000

de la casa consistorial

de Cogua, con

reforzamiento en

maderas confinantes.

Fuente:

elaboración propia

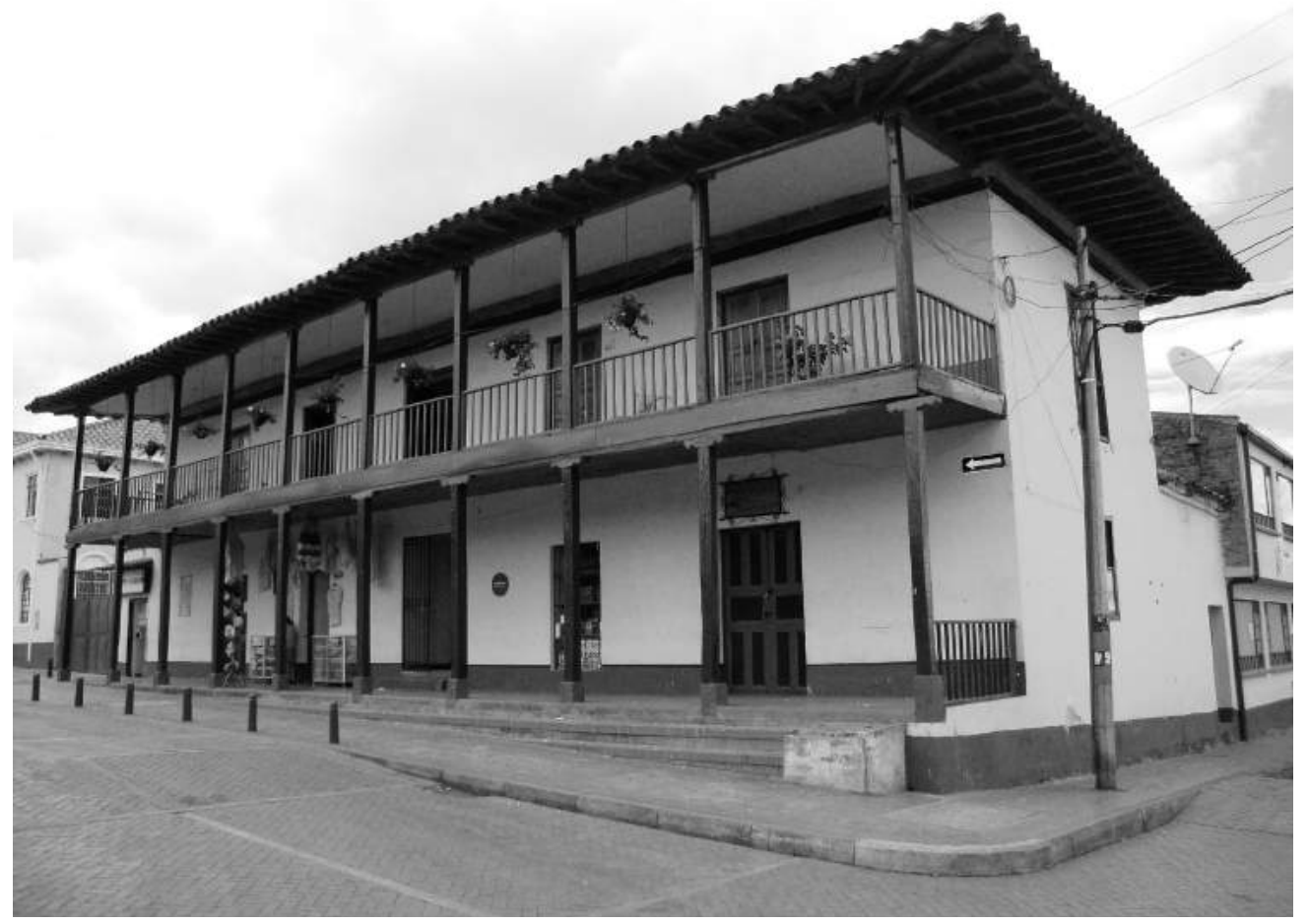

Figura 31:

Esfuerzos en los muros ( $\mathrm{kPa}$ ) de la Casa Cogua, con reforzamiento en maderas confinantes.

Fuente:

elaboración propia

que permite que se puedan realizar sin problemas las regatas para la ubicación de los elementos de reforzamiento, las cuales luego serán pañetadas con mortero de cal y arena, de tal forma que el refuerzo no queda visible y la casa se vea como en su estado natural.

\section{Conclusiones}

Las casas consistoriales de la época colonial en Cundinamarca tienen una tipología definida que se observa repetidamente en los diferentes municipios del departamento. En la gran mayoría de ellos, sus pobladores no tienen conocimiento de la connotación histórica que tiene este inmueble, razón por la cual no se vela por su conservación.

El reforzamiento con maderas confinantes de la casa consistorial del municipio de Cogua es una alternativa para garantizar la preservación del inmueble desde el punto de vista de resistencia sísmica.

Aunque el reforzamiento de la casa Cogua es importante para garantizar su preser- 
vación ante un movimiento sísmico, existen otros factores que se deben tener en cuenta en la conservación de la edificación, entre ellos, la realización de un estudio fitosanitario para determinar el estado de la maderas del entrepiso y cubierta. También es aconsejable disminuir el peso de la cubierta, así como reparar los pilares de madera que se encuentran deteriorados.

Hacen falta estudios más detallados de cómo fue la evolución de este tipo de edificaciones desde el punto de vista constructivo.

\section{Referencias}

AIS, Asociación Colombiana de Ingeniería Sísmica (2005). Manual para la rehabilitación de viviendas construidas en adobe y tapia pisada.

Banco de la República (1991). Groot y el periodismo crítico (cap. 6). José Manuel Groot: (1800-1878). Bogotá: Editorial Banco de la República. Recuperado en http://www. banrepcultural.org/blaavirtual/todaslasartes/ groot/groot3d.htm
Gutiérrez, R. (1921). Monografías (tomo l). Bogotá: Biblioteca de Historia Nacional, Imprenta Nacional.

Gutiérrez, R. (1990). Cabildos y ayuntamientos en América. México: Tilde Editores. Universidad Autónoma de Azcapotzalco.

Groot, J. M. (2009). Por la Sabana de Bogotá y otras historias (pp. 13-39). Bogotá: Alcaldía Mayor de Bogotá, Secretaría, Distrital de Cultura, Recreación y Deporte, Secretaría de Educación del Distrito, Fundación Gilberto Alzate Avendaño.

Hernández, A. (2002). Las instituciones municipales en Colombia, El municipio, célula de descentralización y democracia. Credencial Historia, 147.

Hernández, M. C. (2013). Josefa Ortiz de Domínguez, personaje clave en Historia y Humanidades. Recuperado en http://suite101.net/ article/josefa-ortiz-de-dominguez-personaje-clave-a12333

Hettner, A. (1976). Viajes por los Andes colombianos. (1882-1884) La ciudad, fundación y nombre. Bogotá: Editorial Banco de la República.

Holton, I. F. (1981). La nueva granada: veinte meses en los Andes. Bogotá: Editorial Banco de la República.

Ibarra, J. A. (2003). Mercado, elite e institución: el Consulado de comercio de Guadalajara y el control corporativo de las importaciones en el mercado interno novohispano. Hausberger, B., lbarra, A. (ed.) Comercio y poder en América Colonial. Los Consulados de comerciantes, siglos XVII-XIX. México-Frankfurt: Biblioteca lbero-Americana 93/Vervuert e Instituto Mora, 145-170.

López, J. M. (2006). Los fueros y ordenanzas medievales: embrión del gobierno de los cabildos coloniales hispanoamericanos. Historia, Instituciones, Documentos, 33, 339-363.

López, C., Ruiz, D., Jerez, S., Quiroga, P., Uribe, J. (2007). Rehabilitación sísmica de muros de adobe de edificaciones monumentales mediante tensores de acero. Apuntes, 20(2), 304-317.

Mayorga, F. (2002). El cabildo, institución vertebradora de la vida municipal. Credencial Historia, 147. Recuperado en http://www. banrepcultural.org/blaavirtual/revistas/credencial/marzo2002/elcabildo.htm 
Meli, R., Hernandez, O., Padilla, M. (1980). Strengthening of Adobe Houses for Seismic Action. Proceedings, 7th World Conf. on Earthquake Engineering, 4, Turkish National Committee on Earthquake Engineering. lstanbul, Turkey.

Moreno y Marrero, A. C. (2008). La aportación económica de la colonia guiense de $\mathrm{La} \mathrm{Ha-}$ bana para la construcción del puente del Barranco de las Garzas (1857-1866). Recuperado en http://www.infonortedigital.com/ publicaciones/docs/103.pdf

Montoya, C. (2007). Palabra, imagen y poder. Iconografía de las Casas Capitulares sevillanas para las fiestas de proclamación de Carlos IV. IC Revista Científica de Información y Comunicación, 4, 253-271.

Navarro, P., Vargas, J., y Beas, M. (2009). Seismic Retrofitting Guidelines for the Conservation of Doctrinal Chapels on the Oyón Highlands in Perú. Proceedings of the Getty Seismic Adobe Project 2006 Colloquium.

NSR-10. Norma Colombiana de Construcción Sismo Resistente. Asociación Colombiana de Ingeniería Sísmica. Ministerio de Ambiente, Vivienda y Desarrollo Territorial.

RAE, Real Academia Española de la Lengua (1791). Diccionario de Autoridades (Tomo 11). Disponible en: http://rae.es/

Rivas, M. (1972). Los trabajadores de tierra caliente (capítulo VII, parte 3). Bogotá: Ediciones del Banco Popular.

Rubio, A. (2006). El archivo del cabildo colonial antecedentes históricos. Historia y Espacio, 27. Recuperado en http://historiayespacio.com/images/Contenido/pdf/revista.27/ Rev\%2027\%20-\%20E1\%20Archivo\%20 del\%20Cabildo\%20Colonial.\%20Antecedentes\%20Historicos.PDF

Ruiz, D., López, C. y Rivera, J. (2012). Propuesta de normativa para la rehabilitación sísmica de edificaciones patrimoniales. Apuntes, 25(2), 226-239.
Sánchez, 1. D. (2003). Territorio y urbanismo borbónicos. Las nuevas poblaciones durante el siglo XVIII en el Perú colonial. Arquitextos, 16, 42-46.

Solano, S. P. (2009). Informe Anual del Gobernador de la Provincia de Barranquilla [1870]. Memorias. Revista Digital de Historia y Arqueología desde el Caribe, 6(10), 12.

Solano, S. P. (2010). Del espacio portuario a la ciudad portuaria - los puertos del Caribe colombiano como espacios polifuncionales en el siglo XIX. Varia História , 26(44). Recuperado en http://www.scielo.br/scielo.php?script=sci_arttext\&pid =S0104-87752010000200012.

Suárez, F. (2003). Proyecto de gestión para restaurar la casa consistorial en Guaduas Cundinamarca. Tesis, Universidad del Rosario.

UNEsco (2003). Bam And-E-Bam, Iran. Earth Institute. Unesco Chair Esarthein Architecture. recuperado en: http://www.earth-auroville. com/bam_and_arg_e_bam_en.php

Vigo, A. (1995). El arquitecto benedictino Fray Placido Caamiña y su proyecto de casas consistoriales y cárceles para la ciudad de Ferrol (1791). Cuadernos de estudios Gallegos (tomo 42), 107, 273-312.

Wyrobisz, A. (1980). La ordenanza de Felipe 11 del año 1573 y la construcción de ciudades coloniales españolas en la América. Estudios Latinoamericanos, 7, 11-34.

Yamín, L., Rodríguez, A., Fonseca, L., Phillips, C., Reyes, J. (2003). Comportamiento sísmico y alternativas de rehabilitación de edificaciones en adobe y tapia pisada con base en modelos a escala reducida ensayados en mesa vibratoria. Revista de Ingeniería, 18, 175-190.

Yamín, L., Phillips, C., Reyes, J., Ruiz, D. (2007). Estudios de vulnerabilidad sísmica, rehabilitación y refuerzo de casas en adobe y tapia pisada. Apuntes, 20(2), 286-303. 